\title{
Self-organization of quasi-two-dimensional turbulence in stratified fluids in square and circular containers
}

\section{Citation for published version (APA):}

Maassen, S. R., Clercx, H. J. H., \& Heijst, van, G. J. F. (2002). Self-organization of quasi-two-dimensional turbulence in stratified fluids in square and circular containers. Physics of Fluids, 14(7), 2150-2169.

https://doi.org/10.1063/1.1480263

DOI:

$10.1063 / 1.1480263$

Document status and date:

Published: 01/01/2002

\section{Document Version:}

Publisher's PDF, also known as Version of Record (includes final page, issue and volume numbers)

\section{Please check the document version of this publication:}

- A submitted manuscript is the version of the article upon submission and before peer-review. There can be important differences between the submitted version and the official published version of record. People interested in the research are advised to contact the author for the final version of the publication, or visit the $\mathrm{DOI}$ to the publisher's website.

- The final author version and the galley proof are versions of the publication after peer review.

- The final published version features the final layout of the paper including the volume, issue and page numbers.

Link to publication

\section{General rights}

Copyright and moral rights for the publications made accessible in the public portal are retained by the authors and/or other copyright owners and it is a condition of accessing publications that users recognise and abide by the legal requirements associated with these rights.

- Users may download and print one copy of any publication from the public portal for the purpose of private study or research.

- You may not further distribute the material or use it for any profit-making activity or commercial gain

- You may freely distribute the URL identifying the publication in the public portal.

If the publication is distributed under the terms of Article 25fa of the Dutch Copyright Act, indicated by the "Taverne" license above, please follow below link for the End User Agreement:

www.tue.nl/taverne

Take down policy

If you believe that this document breaches copyright please contact us at:

openaccess@tue.nl

providing details and we will investigate your claim. 


\title{
Self-organization of quasi-two-dimensional turbulence in stratified fluids in square and circular containers
}

\author{
S. R. Maassen, H. J. H. Clercx, and G. J. F. van Heijst \\ Fluid Dynamics Laboratory, Department of Physics, Eindhoven University of Technology, PO Box 513, \\ 5600 MB Eindhoven, The Netherlands
}

(Received 9 January 2002; accepted 1 April 2002; published 23 May 2002)

\begin{abstract}
Laboratory experiments on decaying quasi-2D (two-dimensional) turbulence have been performed in stratified fluids in both square and circular containers. The turbulence was generated by towing an array of vertical cylinders through the container, which was filled with either a two-layer or a linearly stratified fluid. By varying the grid configuration a different amount of angular momentum could be added to the initial flow. The evolution of the flow was visualized by $2 \mathrm{D}$ particle tracking velocimetry. The observed decay scenario has been investigated with emphasis on the evolution of the kinetic energy and the enstrophy of the horizontal flow, vortex statistics and the angular momentum of the flow. In particular it is shown that the experiments in both the square and the circular container support the observations from numerical simulations of decaying 2D turbulence in bounded domains with no-slip walls. Two striking examples are the experimental observation of the spontaneous spin-up phenomenon (in the square-container experiments) and the confirmation that the angular momentum of the flow in the circular-container experiment is better conserved than the total kinetic energy of the flow. The role of the initial nonzero net angular momentum on the decay of quasi-2D turbulence is investigated for both geometries and indications for an acceleration of the self-organization process are presented. (C) 2002 American Institute of Physics.
\end{abstract}

[DOI: $10.1063 / 1.1480263$ ]

\section{INTRODUCTION}

Recently, numerical computations of decaying 2D turbulence in a circular geometry with rigid boundaries by $\mathrm{Li}$ and Montgomery ${ }^{1}$ have revealed some remarkable results. First, these computations showed that, compared to previous simulations on doubly periodic domains, ${ }^{2}$ the presence of rigid (no-slip or stress-free) boundaries alters the decay scenario dramatically. This influence was also observed in a separate numerical study of decaying 2D turbulence on a square domain with rigid boundaries by Clercx et al. ${ }^{3}$ Second, it was observed that, in the circular case with no-slip boundaries, the shape of the final structure depends on whether or not the initial state contained a net nonzero angular momentum. This computer solution of a fundamental fluid dynamics problem suggested - perhaps for the first time - an experiment, which then confirmed this new physical phenomenon. ${ }^{4}$ In the present paper this approach is continued: An experimental study of decaying, quasi-2D turbulence in stratified fluids in square and circular containers is performed and the results are compared with the numerical simulations of decaying $2 \mathrm{D}$ turbulence in similar geometries (with no-slip boundaries).

In the past two or three decades, numerical and experimental studies on 2D turbulence revealed that the process of self-organization occurs by means of many complicated vortex interactions, such as the merging phenomenon described by Matthaeus and Montgomery, who reported for the first time on large-scale convection cells in a NavierStokes simulation with periodic boundary conditions, ${ }^{5}$ by McWilliams ${ }^{6,7}$ and by Santangelo et al. ${ }^{8}$ In most numerical studies it was assumed that the vortices are allowed to move freely on an infinite or periodic plane, and that they are not hampered by any physical boundary. On the other hand, laboratory experiments are generally performed in containers with finite dimensions, which are bounded by impermeable, solid walls. In this paper, it is described how the presence of such physical boundaries influences the self-organization process in (quasi-)2D turbulent flows. What happens when the vortices collide with the wall? How does the geometry of the container influence the shapes of vortices that "survive" the self-organization? In particular, much attention is paid to the effects of viscous boundary layers necessary to satisfy the no-slip boundary conditions at the container wall. Previous numerical studies have shown that these boundary layers play an active role in the flow dynamics. ${ }^{9,10}$

From a few numerical studies we have seen that viscous boundary layers play an important role in the decay of $2 \mathrm{D}$ turbulence on a square domain with no-slip boundaries. ${ }^{11-13}$ The time evolution of these flows differs essentially from the evolution of 2D turbulence with stress-free or doubly periodic boundary conditions. In this paper it will be shown that viscous boundary layers also dominate the flow evolution of laboratory experiments in stably stratified fluids. First, we describe a series of experiments performed in a square container. The results are compared with the numerical results obtained by Clercx et al. ${ }^{11}$ Secondly, the results of experiments in a container with a circular wall are presented. The results of these experiments are compared with numerical simulations of decaying 2D turbulence on a circular domain with no-slip boundaries performed by Li and Montgomery ${ }^{1}$ 
and Li, Montgomery, and Jones ${ }^{14,15}$ (henceforth referred to as LMJ). In all laboratory experiments, an initially smallscale turbulent flow is created by stirring the fluid with a grid of vertical rods. Similar experiments were performed earlier by Flór in circular, square and rectangular containers. ${ }^{16}$ However, in these experiments the forcing was carried out by hand, and the initial condition of the flow was therefore not well-defined.

Much attention is paid to the time evolution of the net angular momentum of the flow. In the simulations performed by LMJ it was observed that, in the circular case with no-slip boundaries, the shape of the final structure depends on the amount of net angular momentum in the initial state. Computations with a significant amount of net angular momentum in the initial flow field revealed that the flow always evolves towards one large central vortex, whereas a rather unsteady "dipolar," or even "quadrupolar" structure emerges in computations with a negligible amount of net angular momentum in the initial state. Furthermore, the angular momentum appeared to be a more slowly decaying quantity than the total kinetic energy. In square containers the net angular momentum behaves differently. It was shown recently with numerical experiments ${ }^{3}$ that the sudden growth of this quantity reflects the spontaneous spin-up of a turbulent flow with an initially zero net angular momentum. This growth of the net angular momentum is accompanied by the formation of a rotating tripolar structure or a domain-filling monopolar vortex. After spontaneous spin-up has occurred, the net angular momentum decays slowly compared to the decay of kinetic energy. The presence of spontaneous spin-up of the flow in the square and circular container, respectively, will be discussed.

The laboratory experiments are focussed on three main issues: (i) What is the influence of the initial net angular momentum on the self-organization of the flow in square and in circular containers? (ii) Which differences are observed between experiments in a square container and those in a circular container, and is it possible to explain the observations? (iii) Do the qualitative evolution of the net angular momentum and the spatial structure of the quasi-stationary states observed in the experiments (in square as well as in circular containers) confirm the results of numerical simulations on decaying 2D turbulence with no-slip boundaries? These questions clearly indicate the somewhat different approach compared with the studies performed by Yap and van Atta ${ }^{17}$ and by Fincham et al. ${ }^{18}$ Yap and van Atta ${ }^{17}$ focused on the velocity correlations, length scales, and velocity and vorticity spectra of stratified turbulence. Additionally, the presence of an inverse energy cascade and the energy dissipation of stratified turbulence is discussed in that paper. Fincham et al. ${ }^{18}$ were concerned with the energy dissipation and the three-dimensional structure of stratified turbulence. Both studies did not take into account the role of the no-slip boundaries and the geometry of the container.

Many complicated processes play a role in stratified turbulence, such as the emergence of internal waves and the formation of layered vortex structures at different heights in the stratification, which are still poorly understood. ${ }^{17-21}$ These experimental difficulties should be kept in mind while interpreting the observations in present investigation (for a discussion of these issues in relation with present experiments, see Maassen ${ }^{22}$ ). The phenomena observed in purely 2D numerical simulations (e.g., the occurrence of spontaneous spin-up of the flow) can therefore only be investigated qualitatively in the laboratory experiments discussed below.

\section{EXPERIMENTAL SET-UP}

The laboratory experiments described in this paper were performed in perspex containers filled with a stably stratified fluid. Two different containers have been used: A square container with dimensions $100 \times 100 \times 30 \mathrm{~cm}^{3}$ (length $\times$ width $\times$ depth) and a circular container with radius $R=46 \mathrm{~cm}$ and depth $30 \mathrm{~cm}$. In the experiments the container-in-use was filled with either a two-layer stratification or a linear stratification. The two-layer stratification consists of a lower layer of salt water (with a density $\rho_{2}$, varying between 1.08 and $1.12 \mathrm{~g} \mathrm{~cm}^{-3}$ ) and a layer of fresh (tap) water on top (density $\rho_{1} \approx 1.00 \mathrm{~g} \mathrm{~cm}^{-3}$ ). Due to mixing introduced during the filling of the container and due to molecular diffusion of salt in water, an interfacial layer (with a depth of typically a few centimeters) is established at the interface between those layers. This interfacial layer thickens slowly, on a time scale of days. The average buoyancy frequency $N$ in the interfacial layer is given by

$$
N^{2}=-\frac{g}{\rho_{0}} \frac{\rho_{1}-\rho_{2}}{h},
$$

with $g$ the gravitational acceleration, $h$ the thickness of the interfacial layer and $\rho_{0}=\left(\rho_{1}+\rho_{2}\right) / 2$ the average density within this layer. In some other experiments a linear vertical density gradient was created by filling the container slowly from beneath using the so-called two-bucket method. ${ }^{23}$ The buoyancy frequency is defined in a similar way as in (1) with $\rho_{1}$ and $\rho_{2}$ the density of the upper and lower layer, respectively, and $h$ the total fluid depth.

In order to create an initially disordered flow, the stratified fluid was forced by towing a grid of vertical bars from one side of the container to the opposite side (similar experiments were performed, for instance, by Lin and Pao, ${ }^{19}$ Yap and van Atta $^{17}$ and Fincham et al. ${ }^{18}$ ). The experimental arrangement is schematically drawn in Fig. 1. A perspex container filled with a stratified fluid (A) is placed on a table, in the center of a mechanical construction of aluminum tubes (B). The two longer sides of this frame are connected by a solid bar (C), which can move in one direction along the aluminum tubes. To produce a quasi-turbulent initial flow, a grid of thin parallel, vertical cylindrical rods (each with diameter $d=3 \mathrm{~mm}$ ) (D) is mounted on the horizontal bar (C) and towed from one side of the container to the opposite side. The bar and the grid are driven by an electric motor (E) mounted on one of the short ends of the frame. Speed and displacement of the grid are adjusted on a control panel (F). After the forcing has stopped, the grid is removed by vertically lifting it out of the fluid. In order to illuminate the flow at a certain height in the fluid, fluorescent strip lights $(\mathrm{G})$ are mounted on the two longer sides of the mechanical construction. Furthermore, a CCD camera (PAL 25 frames s ${ }^{-1}$, Cohu- 


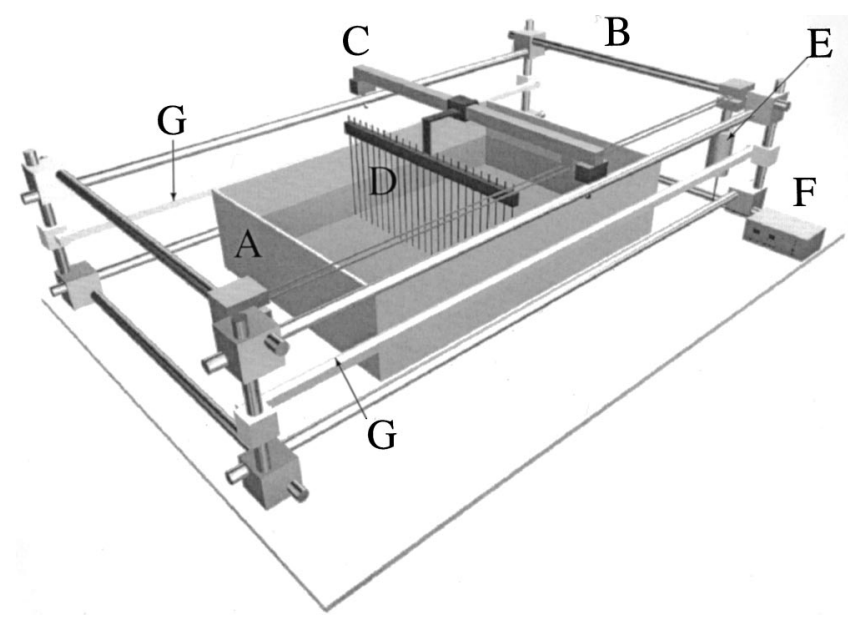

FIG. 1. Schematic picture of the experimental set-up. A: perspex container, B: aluminum frame, C: movable solid bar, D: grid, E: electric motor, F: control panel, G: fluorescent strip lights.

4910) is mounted above the container and connected with a video tape recorder. With a personal computer and a framegrabber the images are digitized to an image resolution of $512 \times 512$ pixels and 8 bit gray level resolution enabling quantitative flow analysis.

An important parameter in the present experimental study is the net angular momentum of the initial flow, which can be controlled by a suitable arrangement of rods in the grid. In each experiment, the configuration of the grid is represented by a series of $y$-coordinates (defined in Fig. 2) corresponding to the positions of the rods. The grid configurations used in the present experiments performed in square and circular containers are listed in the Appendix. Using the drag force exerted by the fluid on each rod, the amount of net angular momentum $L_{0}$ generated in the fluid by a certain grid can be calculated in advance. The drag force exerted on a cylindrical rod with diameter $d$ and length $l$ moving with constant speed $V$ through a quiescent ambient fluid with density $\rho$ can serve as a good estimate (assuming negligible contributions of nonuniformity of the background flow), and is given by

$$
F_{D}=\frac{1}{2} C_{D} \rho V^{2} l d,
$$

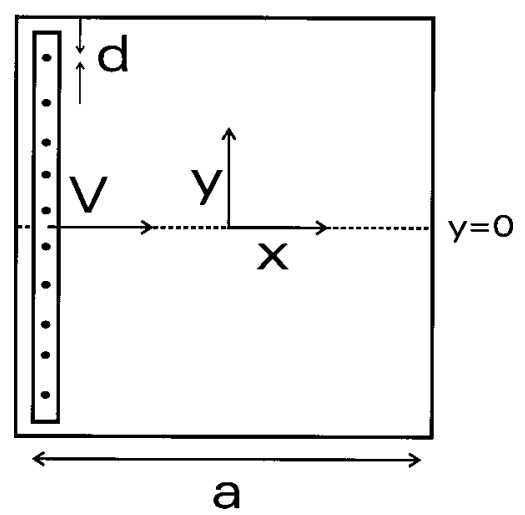

FIG. 2. Top view of the forcing mechanism. A grid of cylindrical rods (each with diameter $d$ ) moves with constant speed $V$ over a distance a along the $x$ axis. where the appropriate value for the drag coefficient $C_{D}$ is obtained from the experimental data collected by Blevins. ${ }^{24}$ Since the rods move parallel and with constant speed, the angular momentum $L_{\text {rod }}$ induced by one rod (per unit length and per unit density), defined with respect to the center of the domain, is proportional to the torque $T$ exerted by this force

$$
L_{\mathrm{rod}}=\frac{1}{\rho l} \int_{0}^{\tau} T d t=\frac{F_{D} y \tau}{\rho l}=\frac{F_{D} y a}{\rho l V}=\frac{1}{2} C_{D} \text { Vyad, }
$$

where $t$ is the time and $\tau$ the time duration of the forcing. The total displacement $a$ is defined in Fig. 2. Since the right-hand side of Eq. (3) is proportional to $y$, the net angular momentum $L_{0}$ induced on the fluid by the grid can be calculated by adding up the contributions of all rods. Note that, in this calculation, the contribution of a rod at position $y$ exactly cancels the contribution of a rod at position $-y$. Using a grid configuration that is symmetric with respect to the line $y$ $=0$, therefore, results in an initial flow with zero net angular momentum $\left(L_{0}=0\right)$. In order to avoid such a symmetry, an initial flow with $L_{0}=0$ may be created by using a nonsymmetric grid, in which the rods are arranged such that the sum of their $y$-coordinates vanishes (see, for example, configurations $\mathrm{S} 2$ and $\mathrm{C} 2$ in the Appendix).

Although the stratified fluid is forced over its whole depth, we will only consider the flow evolution at a certain level in the interface between the two layers of the stratification. Underneath and above this interface, the initial threedimensional (3D) motions induced by the grid show a rapid decay, whereas within the interfacial layer, the planar, quasi-2D flow decays very slowly. This quasi-2D flow is visualized by small polystyrene particles of density 1.04 $\mathrm{g} \mathrm{cm}^{-3}$, a few millimeters in size. Since the density of these particles is smaller than the density of the lower (salty) fluid layer and higher then the upper (fresh) layer, all particles float at approximately the same level in the interfacial layer. The particles are illuminated from the side by fluorescent strip lights (see Fig. 1). During the course of an experiment, the motion of the tracer particles is monitored by a CCD camera mounted at some distance above the container, and the images are stored on a video tape recorder. After the experiment, the Lagrangian trajectories of the tracer particles are determined using the software package DigImage, developed by Dalziel. ${ }^{25}$ A similar approach is undertaken for experiments in linearly stratified fluids.

The particle tracking algorithm used by DigImage consists of three steps. First, a sequence of images at discrete time intervals is taken from the video tape with a frame grabber. Second, particle positions are determined in each image using certain prescribed criteria for size, shape, and brightness of the particles. Finally, the trajectories of the particles are determined by matching their positions at successive time steps. This latter procedure is based on a costfunction method. ${ }^{25}$ Once the particle trajectories have been obtained, instantaneous velocity fields are calculated by taking local (time) derivatives of the trajectories. This procedure results in a set of nongridded velocity vector fields describing the flow evolution. In the present experiments, typically 1000-1500 velocity vectors were found at each time step. 
TABLE I. Characteristic parameters of the experiments performed in a square container. $\mathrm{Re}^{*}=$ Reynolds number, $L_{0}=$ initial net angular momentum, $N$ =buoyancy frequency. The grid configurations (fourth column) are specified in the Appendix.

\begin{tabular}{cccclc}
\hline \hline $\begin{array}{c}\text { Number of } \\
\text { experiments }\end{array}$ & $\mathrm{Re}^{*}$ & $\left|L_{0}\right|$ & Grid & Stratification & $N\left(\mathrm{rad} \mathrm{s}^{-1}\right)$ \\
\hline 1 & 2000 & 0 & $\mathrm{~S} 1$ & linear & 1.6 \\
1 & 4000 & 0 & $\mathrm{~S} 1$ & linear & 1.6 \\
1 & 5000 & 0 & $\mathrm{~S} 1$ & linear & 1.6 \\
3 & 5000 & 0 & $\mathrm{~S} 2$ & two-layer & 2.7 \\
1 & 2000 & 0.4 & $\mathrm{~S} 3$ & linear & 1.6 \\
1 & 3000 & 0.4 & $\mathrm{~S} 3$ & linear & 1.6 \\
1 & 4000 & 0.4 & $\mathrm{~S} 3$ & linear & 1.6 \\
1 & 2000 & 0.6 & $\mathrm{~S} 4$ & linear & 1.6 \\
1 & 3000 & 0.7 & $\mathrm{~S} 4$ & linear & 1.6 \\
1 & 4000 & 0.7 & $\mathrm{~S} 4$ & linear & 1.6 \\
\hline \hline
\end{tabular}

In order to compute related quantities such as the vorticity field and the stream function, the vector fields are interpolated to a regular grid of $40 \times 40$ mesh points, using cubic splines as interpolation functions. Although in principle the vorticity field can be computed analytically from the spline coefficients, this method gives rise to many spurious errors in the vorticity contour plots, especially when the number of velocity vectors computed in the PTV procedure is large (>900). Therefore, we have chosen to fit the gridded vector fields, obtained in the experiments in the square container, with a doubly truncated series of Chebyshev polynomials. (Spline interpolation has been used for the experiments in the circular tank.) The fitting algorithm is a two-dimensional version of the algorithm proposed by Clenshaw. ${ }^{26}$ It consists of an iterative procedure in which the order of the Chebyshev expansion is gradually increased in both directions. At each iteration step, a least square fit of the original vector field is computed. The iteration stops when the standard deviation of the difference between the original vector field and the fit reaches a minimum. Although the algorithm can be applied directly to the nongridded data obtained by PTV, we have found that more accurate results are obtained when the data are first interpolated to a regular grid.

\section{THE SQUARE CONTAINER}

First we describe a series of experiments that are performed in a square container (horizontal dimensions 100 $\times 100 \mathrm{~cm}^{2}$ ). Three experiments were performed in a twolayer fluid, all others in a linear stratification. It appears that the type of stratification does not have a substantial influence on the decay properties of quasi-2D turbulence. The characteristic parameters of the experiments performed in a square container are listed in Table I.

Let us first briefly summarize the subsequent processes that take place in a decaying stably stratified turbulent flow, following the models of Riley et al. ${ }^{20}$ and Lilly. ${ }^{21}$ We assume that the vortices emerging in the wake behind the grid have a characteristic velocity in the order of the speed of the grid (which is typically $V=15 \mathrm{~cm} \mathrm{~s}^{-1}$ ) and a characteristic length scale $\lambda$ comparable with the diameter $d$ of the bars in the grid (typically $0.3 \mathrm{~cm}$ ). In the linearly stratified fluid (or in the interfacial layer of the two-layer fluid), the initial Froude number of the fluid, defined as $\mathrm{Fr}=V /(N \lambda)$, is $\mathrm{Fr} \approx 25$. This means that the buoyancy term in the Boussinesq equation can be neglected and the initial flow in the linear stratification can be considered as approximately 3D. However, as the kinetic energy associated with these $3 \mathrm{D}$ eddies is rapidly dissipated, the Froude number drops to values of order one within a few seconds (see, e.g., Riley et al. ${ }^{20}$ and Riley and Lelong $^{27}$ ). In the first measured velocity field (usually $10 \mathrm{~s}$ after the forcing has stopped), the average horizontal velocity is approximately $1 \mathrm{~cm} \mathrm{~s}^{-1}$ and the horizontal length scale of the vortices is typically a few $\mathrm{cm}$, yielding a Froude number $\mathrm{Fr} \approx 0.1$. (It would be more useful to compute the Froude number based on the vertical velocity and the vertical length scale, but this information could not be obtained nor estimated from the experiments.) This low Froude number indicates that the effects of stratification dominate the flow evolution. In this regime, the turbulent flow collapses towards a quasi-2D flow, in which velocities in the vertical direction are negligible compared with the typical velocities in the horizontal plane. ${ }^{20,27}$ This quasi-2D flow is characterized by the formation of pancake-like vortices, which show a slow decay. Simultaneously, part of the kinetic energy of the initial 3D motions is converted into potential energy, thus leading to the generation of internal waves. Although the interaction between internal waves and quasi-2D flow is not completely understood, ${ }^{27}$ we assume that these interactions are negligible for the present analysis, which from now on only considers the quasi-2D vortex flow.

In order to facilitate a comparison of our experiments with the numerical simulations presented in Ref. 11, the Reynolds number of the flow is defined as $\operatorname{Re}^{*}=W U / \nu$, with $W$ the half width of the container, $U$ the root-mean-squared velocity of the initial flow field, and $\nu$ the kinematic viscosity of water. [Strictly speaking, the kinematic viscosity $\nu$ of the fluid depends on the salinity of the fluid. However, for the salt concentrations that are used in the present experiments, $\nu$ varies only a few percent. Therefore, we assume that $\nu$ $=10^{-2} \mathrm{~cm}^{2} \mathrm{~s}^{-1}$ (the kinematic viscosity of water at $20^{\circ} \mathrm{C}$ ) in all experiments.] Throughout this section, all variables are made dimensionless by using the length scale $W$ and the eddy-turnover time $W / U$. The value for $U$ is obtained from the first measured velocity field, which usually corresponds to the flow field at 10 seconds after the forcing has stopped. Due to inaccuracies of the particle tracking system and the applied interpolation method, the computed value of $U$ contains an error of approximately 10\%. Furthermore, at this early stage of the evolution, the flow may still be affected by 3D turbulent motions. Altogether, the error in the estimated value of $\mathrm{Re}^{*}$ is of the order of $15 \%$. In the present experiments, $2000 \leqq \mathrm{Re}^{*} \leqslant 5000$. However, in a stably stratified fluid the dissipation of kinetic energy is dominated by vertical shearing ${ }^{17,18}$ and the effective Reynolds number related to the measured quasi-2D flow is therefore much smaller than $\mathrm{Re}^{*}$. The Reynolds number based on the typical towing speed of the grid ( $V \simeq 15 \mathrm{~cm} \mathrm{~s}^{-1}$ ) and the average spacing of the grid rods $(M \simeq 4 \mathrm{~cm})$ yields: $R e=V M / \nu \simeq 6000$. The Reynolds number in the experiments by Yap and van Atta ${ }^{17}$ and 


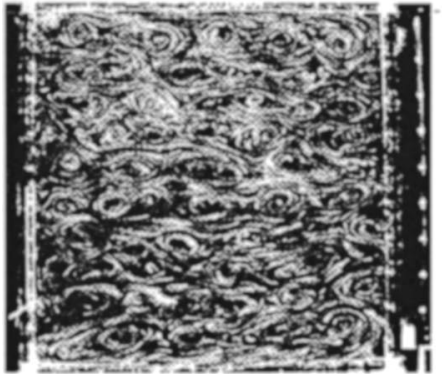

(a) $t=0.2$

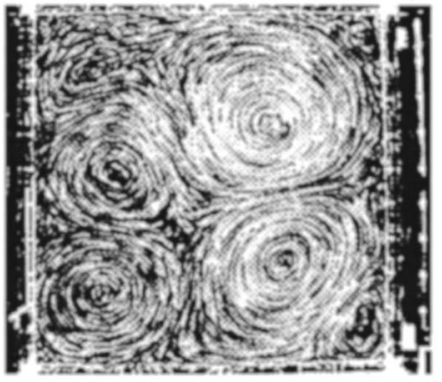

(d) $t=12$

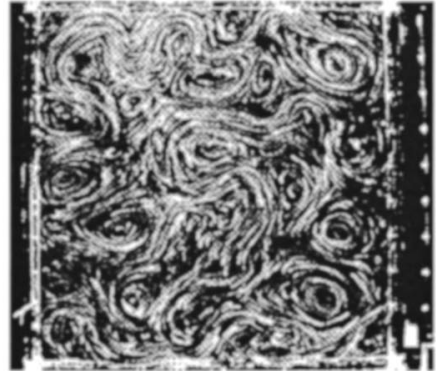

(b) $t=1$

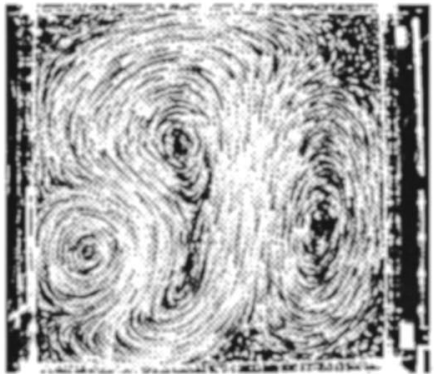

(e) $t=50$

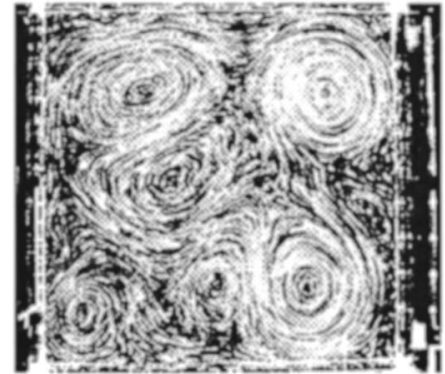

(c) $t=6$

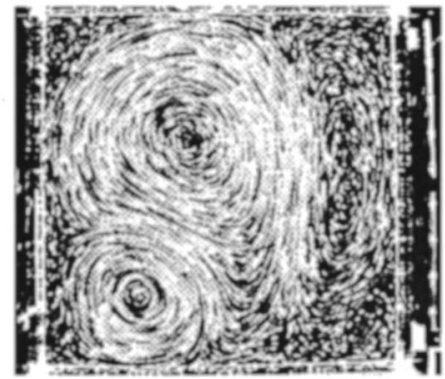

(f) $t=60$

FIG. 3. Streak images of an experiment with $L_{0} \approx 0$ in a square container. The tails of the streaks represent the displacements of tracer particles during an interval of (a) and (b) 0.1, (c) and (d) 0.2, and (e) and (f) 4 dimensionless time units.

by Fincham et al.,${ }^{18}$ defined as $\operatorname{Re}=V M / v$, are $\operatorname{Re} \simeq 5000$ and $700 \leq \operatorname{Re} \leq 12000$, respectively.

The total kinetic energy $E$ is defined as

$$
E(t)=\frac{1}{2} \int_{-1}^{1} \int_{-1}^{1}\left[u^{2}(x, y, t)+v^{2}(x, y, t)\right] d x d y,
$$

with $u$ and $v$ the $x$ and $y$ components, respectively, of the measured horizontal velocity field. The angular momentum of the flow, defined with respect to the center of the container, is

$$
\begin{aligned}
L(t) & =\int_{-1}^{1} \int_{-1}^{1}[x v(x, y, t)-y u(x, y, t)] d x d y \\
& =2 \int_{-1}^{1} \int_{-1}^{1} \psi(x, y, t) d x d y,
\end{aligned}
$$

with the stream function $\psi$ defined as: $u=\partial \psi / \partial y$ and $v=-\partial \psi / \partial x$. On a bounded domain with stationary no-slip walls (thus with zero circulation of the flow in the domain $\mathcal{D}$ ) the time rate of change of $L$ can be expressed as

$$
\frac{d L}{d t}=\frac{1}{\rho} \oint_{\partial \mathcal{D}} p \mathbf{r} \cdot d \mathbf{s}+\frac{1}{\operatorname{Re}} \oint_{\partial \mathcal{D}} \omega(\mathbf{r} \cdot \hat{\mathbf{n}}) d s,
$$

where $\hat{\mathbf{n}}$ is the unit vector normal to the boundary and $d s$ denotes the length of an infinitesimal element $d \mathbf{s}$ of the boundary $\partial \mathcal{D}$.

\section{A. Spatial flow evolution}

The streak images displayed in Fig. 3 indicate the global evolution of an experiment with initially zero net angular momentum in a linear stratification with buoyancy frequency $N \approx 1.6 \mathrm{rad} \mathrm{s}^{-1}$ and $\mathrm{Re}^{*}=5000$. In this experiment we moved the grid from right to left. As a first observation, note that one vortex dominates the final flow structure in this experiment. Figure 3(a) was taken a few seconds after the grid had been removed from the container. The horizontal jets in this photograph are due to a nonuniform distribution of rods in the grid (see the Appendix). The rods were arranged in five groups, each consisting of five rods with mutual distances of $2 \mathrm{~cm}$, and two adjacent groups of rods were separated by a "gap" of about $10 \mathrm{~cm}$ (grid S1). When the grid is towed through the fluid, these gaps result in the backward jets visible in Fig. 3(a). Note that in the square geometry the rods of the grid move parallel to the walls of the container, and the forcing covers the whole area of the fluid.

Figure 4 displays the decay of the total kinetic energy and the total enstrophy of the experiment shown in Fig. 3, normalized by their initial values. Comparing these curves with the decay of kinetic energy observed in numerical simulations with approximately the same value of the Reynolds number, it can be concluded that the quasi-2D experimental flow decays much faster than the purely $2 \mathrm{D}$ simulated flow. The enhanced energy dissipation in the experiments is mainly due to vertical shearing between different layers in the stratification. ${ }^{17,18}$ The decay rates of kinetic energy obtained from laboratory experiments with $\mathrm{Re}^{*} \approx 5000$ are of the same order as those computed in numerical simulations with $R e \approx 1500-2000 .{ }^{11}$ Based on this observation we estimate that, for the present experiments, the decay times of an 


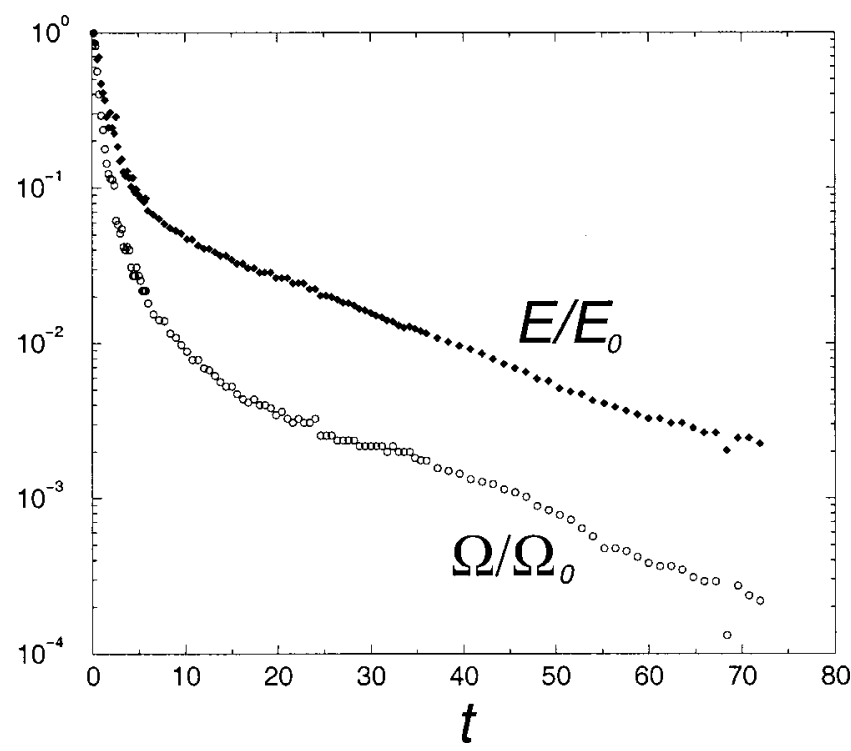

FIG. 4. Normalized kinetic energy $E / E_{0}$ and normalized enstrophy $\Omega / \Omega_{0}$ plotted logarithmically versus dimensionless time for the experiment shown in Fig. 3 (square container, $L_{0} \approx 0$ ).

experiment with Reynolds number $\mathrm{Re}^{*}$ are comparable with the decay times of a numerical simulation with Reynolds number $\mathrm{Re} \approx 0.4 \mathrm{Re}^{*}$. A more extensive discussion of the energy dissipation in stratified grid turbulence is presented in Sec. III B.

Figures 5 and 6 show the time evolution of the vorticity field and the stream function, respectively, of the same experiment as shown in Fig. 3. The vorticity is calculated from the nongridded velocity vectors following the procedure described in Sec. II. The stream function is calculated directly from the interpolated vorticity field by solving the Poisson equation $\nabla^{2} \psi=-\omega$ with $\psi=0$ at the boundaries of the container. In Fig. 5(a) the small vortices induced by the forcing mechanism are clearly ordered in "streets" of vortices of the same sign. Most likely, these vortices are the result of a shear instability in the backwards moving jets, which occur in the gaps of the moving grid. Note that this initial condition is different from the well-known Von Kármán vortex street emerging by shedding of vortices behind a moving cylinder. The vortices that are shed from the cylindrical rods have a size comparable with the diameter of the grid $(3 \mathrm{~mm} \simeq 0.015$ $\mathrm{W}$ ), and are not visible in the displayed pictures. In Figs. 5(b) and 6(a) the ordered structure has disappeared, and the vortices move freely through the domain. Some vortices form dipolar structures, whereas others are torn apart by neighboring vortices. Two opposite-signed vortices in the upper and lower right corners of the domain are considerably stronger than the other vortices [Figs. 5(b) and 5(c)]. These vortices form a dipole which moves from right to left in Figs. 5(d) and 5(e). However, negative vorticity created near the lower boundary gradually rolls up into a new vortex, and a tripolar structure is formed at $t \approx 30$ [Figs. 5(e) and 6(c)]. This tripolar structure rotates about 45 degrees between $t \approx 30$ and $t$ $\approx 50$. For $t \gtrsim 50$ the flow is dominated by viscous decay.

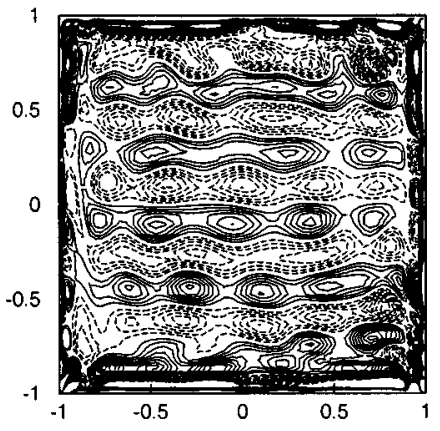

(a) $t=0.2$

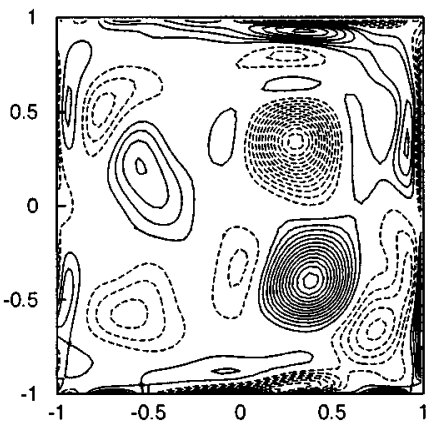

(d) $t=12$

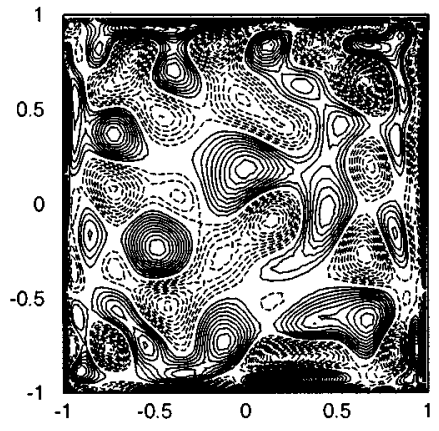

(b) $t=1$

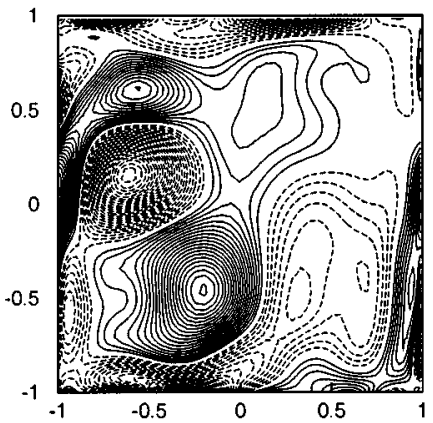

(e) $t=36$

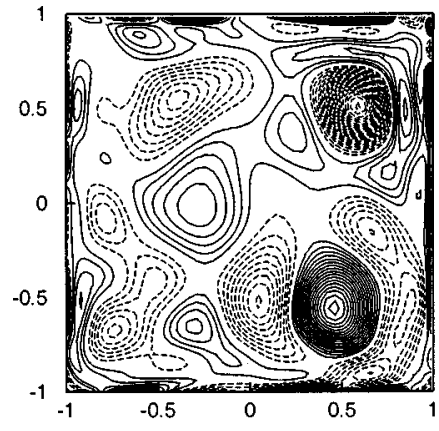

(c) $t=6$

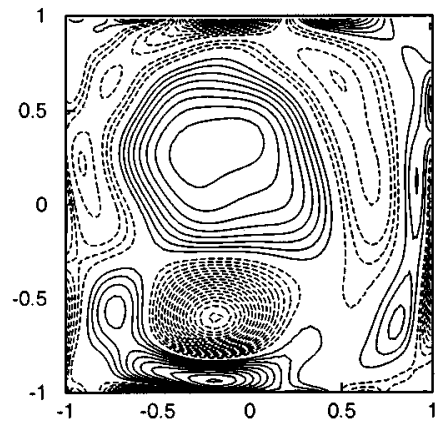

(f) $t=72$

FIG. 5. Vorticity contour plots of the same experiment as in Fig. 3 (square container, $L_{0} \approx 0$ ). Dashed contours represent negative values of vorticity, and solid contours represent positive values. The contour level increment is (a) 5, (b) 1.5, (c) 0.5, (d) 0.45, (e) 0.1, and (f) 0.05 . 


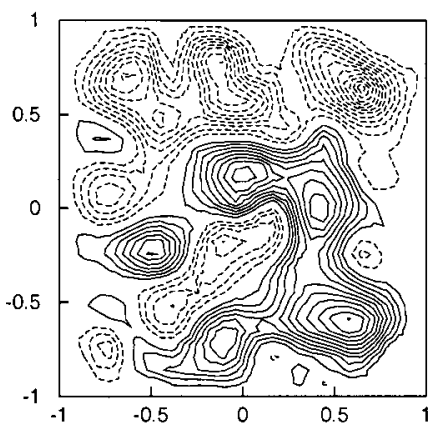

(a) $t=1$

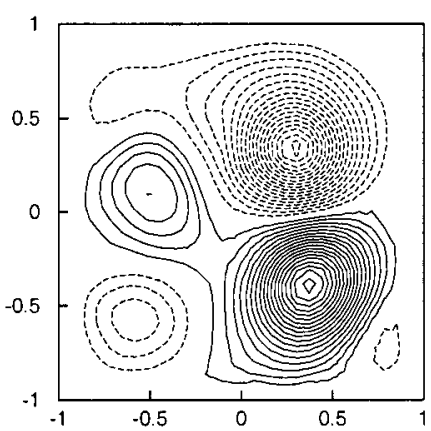

(b) $t=12$

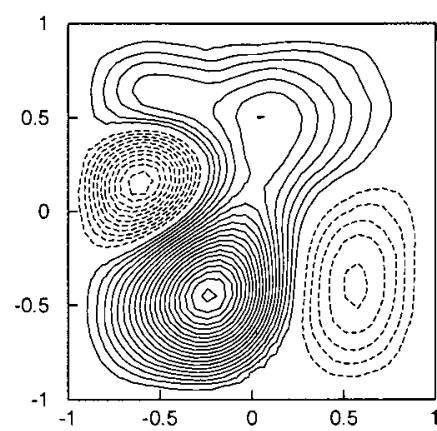

(c) $t=36$

FIG. 6. Stream function contour plots of the same experiment as in Figs. 3 and 5 (square container, $L_{0} \approx 0$ ). Dashed contours represent negative stream function values, and solid contours represent positive values. The contour level increment is (a) 0.02 , (b) 0.008 , and (c) 0.004 .

The formation of a rotating tripolar structure from an initially disordered flow with zero net angular momentum indicates that the flow has acquired a net amount of angular momentum during its decay. This spontaneous spin-up phenomenon has recently been predicted numerically (for $\operatorname{Re} \leqslant 2000$, see Ref. 3, and for $5000 \leqslant \operatorname{Re} \leqslant 20000$, see Ref. 28). Spontaneous spin-up of the flow is demonstrated in Fig. 7 (a), which displays the time evolution of the dimensionless net angular momentum for the experiment shown in Figs. 5 and 6 . The large fluctuations during the first few eddy turnover times are partly due to experimental errors, partly due to the emergence of internal waves, and partly due to the creation and detachment of viscous boundary layers [see also Fig. 7(b) where the large fluctuations for $t \leqslant 20$ are predominantly due to boundary layer detachment]. After about 25 eddy turnover times the angular momentum starts suddenly to increase up to $L=0.08$ at $t \approx 30$. [The net angular momentum of the same amount of fluid with the same energy $E(t$ $=30)=0.02$ is $L_{s b}=0.33$.] This event coincides with the formation of the tripolar structure in Figs. 5(e) and 6(c).

In order to discriminate between the coherent vortex cores and the cascading turbulent background, McWilliams used the Weiss function $Q$, defined as ${ }^{29}$

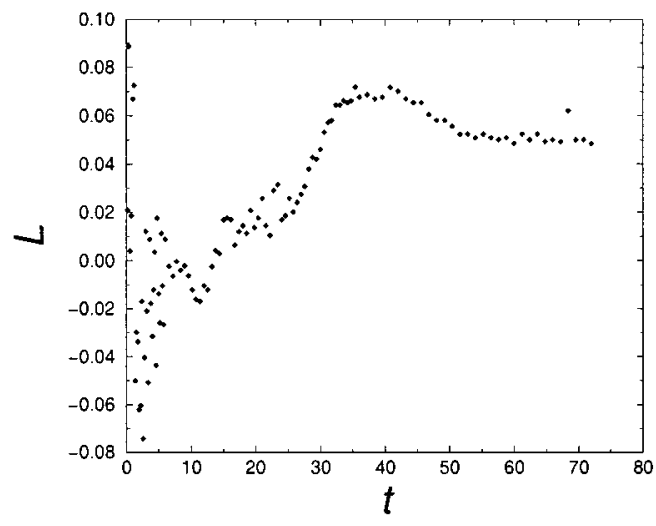

(a) experiment, $R e^{*}=5000$

$$
\begin{aligned}
Q & =S^{2}-\omega^{2} \\
& =\left(\frac{\partial u}{\partial x}-\frac{\partial v}{\partial y}\right)^{2}+\left(\frac{\partial v}{\partial x}+\frac{\partial u}{\partial y}\right)^{2}-\left(\frac{\partial v}{\partial x}-\frac{\partial u}{\partial y}\right)^{2},
\end{aligned}
$$

with $\omega$ the vorticity and $S$ a measure of the rate of strain in a particular area of the flow: $S^{2}=-\operatorname{det}\left(\nabla \mathbf{v}+\nabla \mathbf{v}^{\mathbf{T}}\right)$. Regions with $Q<0$ are rotation-dominated ( $\omega>S$ ) and have an elliptic or neutral character: Two fluid particles initially near each other in an elliptic region will stay close together. In regions where $Q>0$ the flow is strain-dominated $(\omega<S)$ and has a hyperbolic or turbulent character: The distance between two fluid particles in these regions increases exponentially in time. Vortex cores are found in regions with $Q<0$, that are surrounded by rings with $Q>0$. In these latter areas, gradients of vorticity tend to grow and form long thin filaments of vorticity. Therefore, the turbulent enstrophy cascade is most active in regions with maximum $Q$ (in the vicinity of vortices) and is excluded from the vortex cores (in which $Q<0$ ).

In Fig. 8 we have plotted some contours of the Weiss function $Q$ for the experiment shown in Figs. 5 and 6. Snapshots of $Q$ are shown for $t=1,6,12$, and 36. Dashed con-

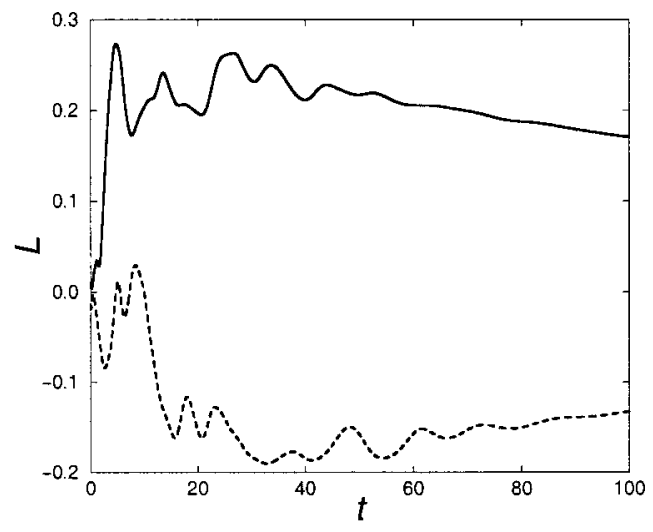

(b) simulations, $R e=2000$

FIG. 7. (a) Dimensionless net angular momentum versus dimensionless time for the experiment shown in Figs. $3-6$ (square container, $L_{0} \approx 0$ ). (b) Angular momentum $L$ plotted versus dimensionless time $t$ for two typical runs with $\operatorname{Re}=2000$ showing spontaneous spin-up. 


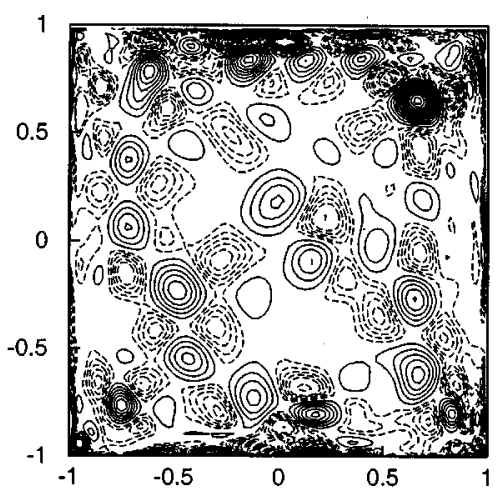

(a) $t=1$

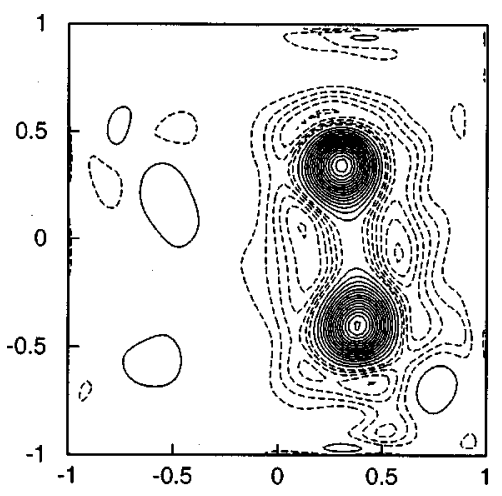

(c) $t=12$

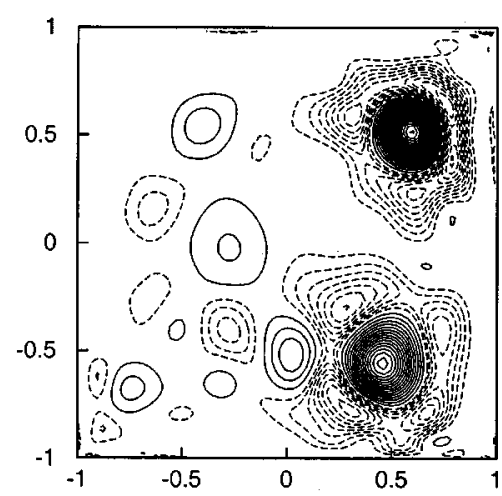

(b) $t=6$

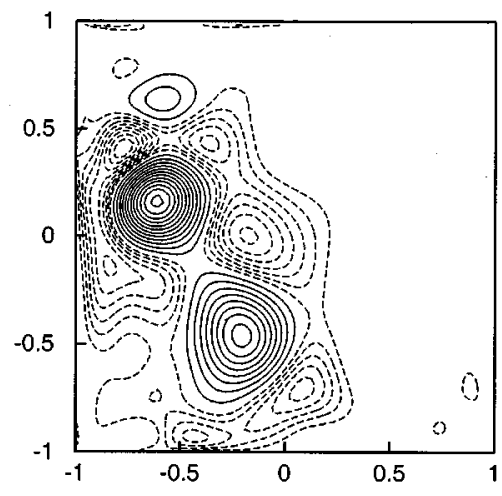

(d) $t=36$
FIG. 8. Weiss function [see Eq. (7)] contour plots of the same experiment as in Figs. 5 and 6 (square container, $L_{0} \approx 0$ ). Dashed contours represent hyperbolic regions $(Q>0)$ and solid contours represent elliptic regions $(Q<0)$. The contour level increment is (a) 25, (b) 2.5, (c) 1.25 , and (d) 0.25 for the dashed contours and (a) 50 , (b) 5 , (c) 2.5 , and (d) 5 for the solid contours. tours represent hyperbolic, or strain-dominated, regions $(Q$ $>0)$, and elliptic, or vorticity-dominated, regions are denoted by solid contours $(Q<0)$. Comparing the pictures of Figs. 5 and 8 at equal times clearly shows that elliptic regions are found in the vortex cores, whereas the viscous boundary layers are always found in strain-dominated regions. This latter property explains the detachment of viscous boundary layers, as is illustrated, for instance, near the lower part of the right wall in Figs. 5(c) and 8(b) and near the upper part of the left wall in Figs. 5(e) and 8(d). Furthermore, the rotation-dominated regions have a circular or elliptical shape. Comparing these observations with the Weiss function contour plots shown in Ref. 11 clearly indicates that, as expected, the (no-slip) experimental flows evolve in a similar way as flows in simulations with no-slip boundary conditions, and that their spatial evolution deviates strongly from simulations with stress-free or with periodic boundary conditions.

\section{B. Ensemble averaging and vortex statistics}

Several experiments with $L_{0} \approx 0$ have been carried out with different values of $\mathrm{Re}^{*}$ (see Table I). First, we have investigated how the strength of the stratification influences the global decay processes of grid turbulence in a square container. Experiments performed by Yap and van Atta ${ }^{17}$ in two-layer fluids showed that a stronger stratification enhances dissipation due to vertical shearing, but it also suppresses vertical motions, thus enhancing the two- dimensionality of the flow at the interface. In addition, Fincham et al. ${ }^{18}$ directly measured the various terms that contribute to the total energy dissipation rate $\dot{E} \equiv d E / d t$ in a linear stratification. These authors showed that $\dot{E} \approx \epsilon_{z}$ $+\epsilon_{2 \mathrm{D}}$, with $\epsilon_{z}$ the dissipation due to vertical shearing of the horizontal velocity, defined by

$$
\begin{aligned}
\epsilon_{z} & =-\frac{1}{\operatorname{Re}} \int_{\mathcal{D}}\left[\left(\frac{\partial u}{\partial z}\right)^{2}+\left(\frac{\partial v}{\partial z}\right)^{2}\right] d x d y \\
& \approx-\frac{2}{\operatorname{Re}} \int_{\mathcal{D}}\left(\frac{\partial u}{\partial z}\right)^{2} d x d y
\end{aligned}
$$

and $\epsilon_{2 \mathrm{D}}$ the dissipation due to horizontal strain, defined by

$\epsilon_{2 \mathrm{D}}=-\frac{1}{\operatorname{Re}} \int_{\mathcal{D}}\left[\left(\frac{\partial u}{\partial y}+\frac{\partial v}{\partial x}\right)^{2}+\left(\frac{\partial u}{\partial x}-\frac{\partial v}{\partial y}\right)^{2}\right] d x d y=-\frac{2 \Omega}{\operatorname{Re}}$

(Note that Fincham et al. ${ }^{18}$ use a different notation.) They concluded that approximately $80 \%$ of the total dissipation is due to vertical shearing $\left(\epsilon_{z}\right)$, whereas $\epsilon_{2 \mathrm{D}}=-2 \Omega / \operatorname{Re}$ accounts for less than $20 \%$ of the total energy dissipation.

Figure 9 shows the average decay of the normalized kinetic energy $E / E_{0}$ [Fig. $\left.9(\mathrm{a})\right]$ and the normalized enstrophy 


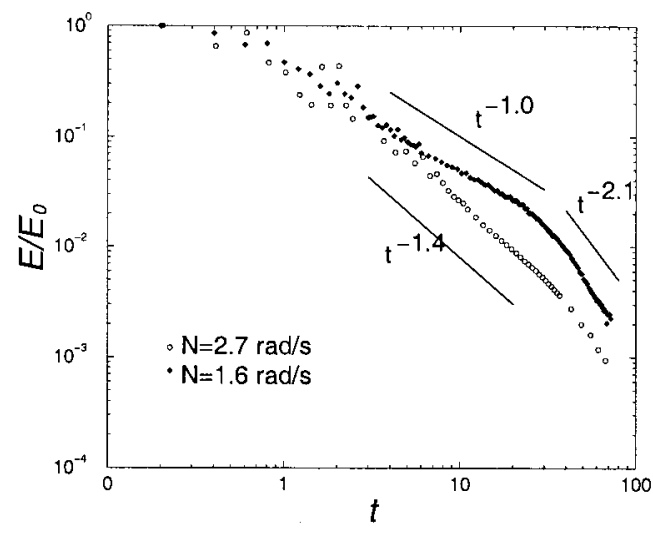

(a)

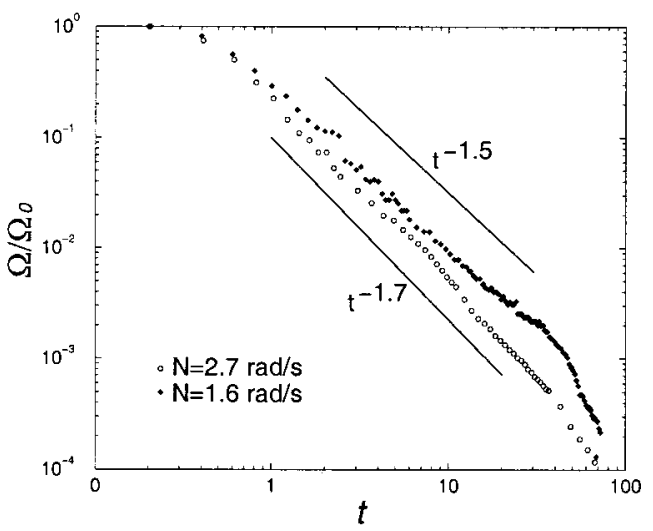

(b)

FIG. 9. Log-log plots of (a) the normalized kinetic energy and (b) the normalized enstrophy, both versus dimensionless time (square container, $L_{0} \approx 0$ ). The open symbols represent ensemble averages obtained from three experiments in a strong stratification $\left(N=2.7 \mathrm{rad} \mathrm{s}^{-1}\right)$; the filled symbols correspond to an experiment in a weak stratification $\left(N=1.6 \mathrm{rad} \mathrm{s}^{-1}\right)$.

$\Omega / \Omega_{0}$ [Fig. 9(b)] of three experiments performed in a stratification with buoyancy frequency $N \approx 2.7 \mathrm{rad} \mathrm{s}^{-1}$ (dots) and one experiment with $N \approx 1.6 \mathrm{rad} \mathrm{s}^{-1}$ (squares), both with $\mathrm{Re}^{*}=5000$. (The experiments with $N \approx 2.7 \mathrm{rad} \mathrm{s}^{-1}$ concerned two-layer stratified fluids and the experiment with $N$ $\approx 1.6 \mathrm{rad} \mathrm{s}^{-1}$ a linearly stratified fluid. The type of stratification does not have a substantial influence on the decay properties of quasi-2D turbulence.) The experimental data for the normalized energy and enstrophy were fitted with power laws $E \propto t^{\alpha}$ and $\Omega \propto t^{\beta}$, respectively. As a result, we found $\alpha=-1.4 \pm 0.2$ and $\beta=-1.7 \pm 0.2$ for the experiments in a strong stratification $\left(N \approx 2.7 \mathrm{rad} \mathrm{s}^{-1}\right)$, and $\alpha=-1.0 \pm 0.1$ and $\beta=-1.5 \pm 0.2$ for the experiment in a weaker stratification $\left(N \approx 1.6 \mathrm{rad} \mathrm{s}^{-1}\right)$. Dissipation of kinetic energy is obviously more effective in experiments with a stronger stratification, as was also found by Yap and van Atta, ${ }^{17}$ whereas the dissipation rates of enstrophy are almost the same in the two cases.

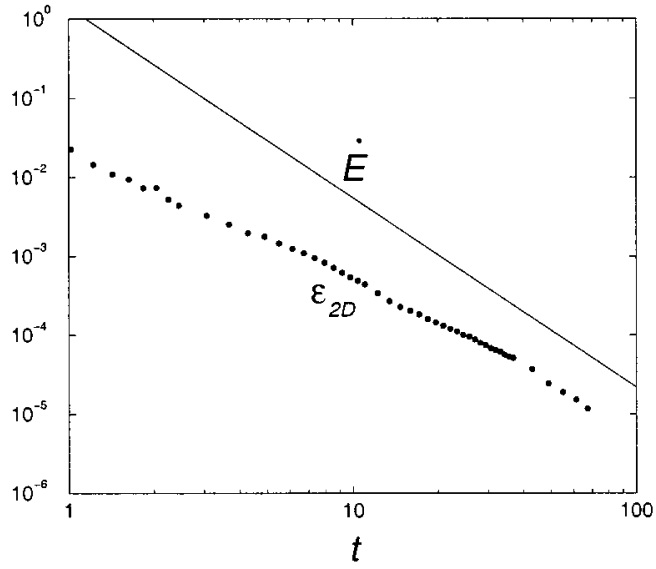

(a)
In Fig. 10 we have plotted the time derivative of the normalized kinetic energy, together with the normalized dissipation rate due to horizontal strain, defined as $\epsilon_{2 \mathrm{D}} / E_{0}$ $=-2 \Omega /\left(\operatorname{Re}^{*} E_{0}\right)$, for the ensemble averages obtained from three experiments in a strong stratification $\left[N=2.7 \mathrm{rad} \mathrm{s}^{-1}\right.$, Fig. 10(a)] and one experiment in a weak stratification $(N$ $=1.6 \mathrm{rad} \mathrm{s}^{-1}$, Fig. 10(b)]. The lines for $\dot{E} / E_{0}$ are estimated from the data for the normalized kinetic energy plotted in Fig. 9(a). For $N=1.6 \mathrm{rad} \mathrm{s}^{-1}$ and $N=2.7 \mathrm{rad} \mathrm{s}^{-1}$ we obtain $\dot{E} / E_{0} \propto t^{-2.0}$ and $\dot{E} / E_{0} \propto t^{-2.4}$, respectively. The normalized values of $\dot{E}$ and $\epsilon_{2 \mathrm{D}}$ plotted in Fig. 10 are of the same order as the values of these quantities obtained by Fincham et al. ${ }^{18}$ Following these authors, we conclude that the energy dissipation in the present experiments is indeed dominated by vertical shear.

Considering all experiments with $L_{0} \approx 0$ in a square container, spontaneous spin-up of the flow was observed in all

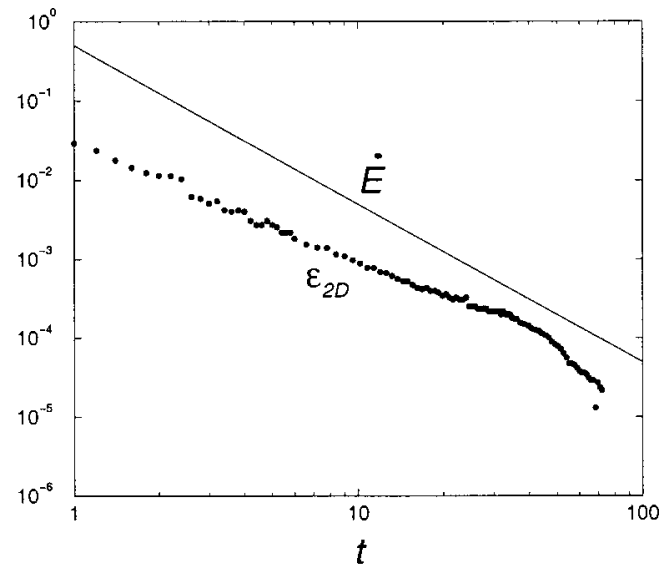

(b)

FIG. 10. Normalized total energy decay rate $\dot{E} / E_{0}$ (continuous lines) and normalized dissipation due to horizontal shear $\epsilon_{2 \mathrm{D}} / E_{0}=-2 \Omega /\left(\mathrm{Re} * E_{0}\right)($ dots) both plotted versus time for (a) the ensemble averages obtained from three experiments in a strong stratification $\left(N=2.7 \mathrm{rad} \mathrm{s}^{-1}\right)$ and $(\mathrm{b})$ one experiment in a weak stratification $\left(N=1.6 \mathrm{rad} \mathrm{s}^{-1}\right)$. The lines for $\dot{E} / E_{0}$ are estimated from the data plotted in Fig. 9(a) (square container, $L_{0} \approx 0$ ). 


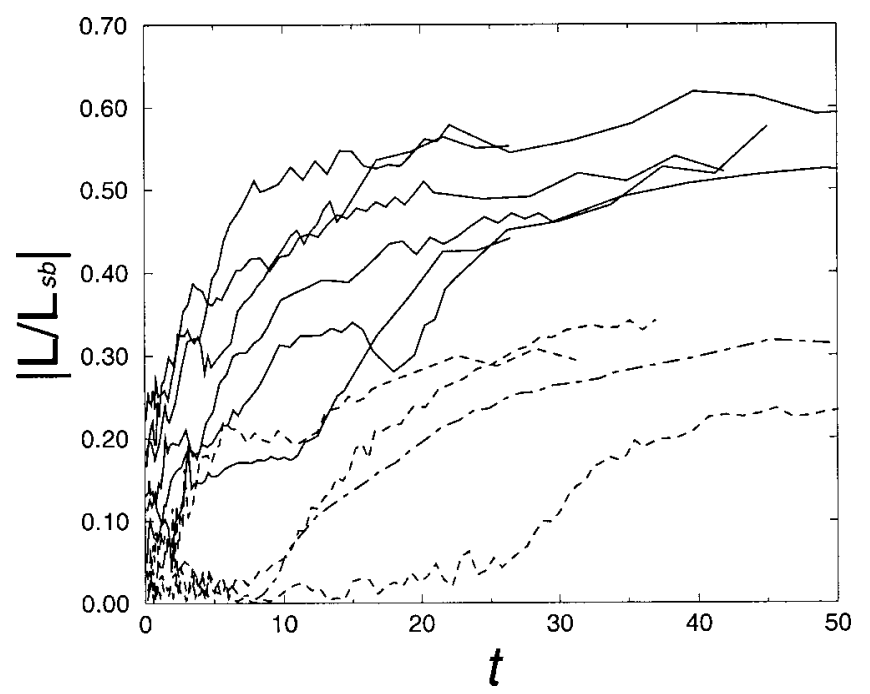

FIG. 11. Normalized net angular momentum $L / L_{s b}(t)$ for several experiments performed in a square container with $L_{0} \approx 0$ (dashed and dasheddotted lines) and $\left|L_{0}\right|>0$ (solid lines). The dashed-dotted line corresponds to an experiment with $N \approx 2.7 \mathrm{rad} \mathrm{s}^{-1}$; dashed and solid lines represent experiments with $N \approx 1.6 \mathrm{rad} \mathrm{s}^{-1}$.

experiments with a weak stratification $\left(N \approx 1.6 \mathrm{rad} \mathrm{s}^{-1}\right)$, and only in one experiment with a stronger stratification $(N$ $\approx 2.7 \mathrm{rad} \mathrm{s}^{-1}$ ). Our interpretation is that in experiments with $N \approx 2.7 \mathrm{rad} \mathrm{s}^{-1}$ the effective Reynolds number decreases so rapidly due to vertical shear, that nonlinear processes such as spontaneous spin-up are suppressed by viscous dissipation. The absolute value of the normalized net angular momentum $\left|L / L_{s b}(t)\right|$ is plotted in Fig. 11 of several experiments with $L_{0} \approx 0$ (dashed and dashed-dotted lines), where $L_{s b}$ is the angular momentum of the same amount of fluid with total kinetic energy $E(t)$ in solid-body rotation. The dasheddotted line corresponds to an experiment with $N$ $\approx 2.7 \mathrm{rad} \mathrm{s}^{-1}$, the dashed lines represent experiments with $N \approx 1.6 \mathrm{rad} \mathrm{s}^{-1}$. (The solid lines correspond to experiments with $L_{0} \neq 0$ which are discussed in Sec. III C.) Although the maximum absolute value of the net angular momentum is generally lower than the spin-up amplitude observed in nu- merical simulations (see Ref. 11, Fig. 8), a moderate increase of angular momentum is observed in all experiments in a square container with $L_{0} \approx 0$.

One would expect that, after performing a large number of experiments, both positive and negative monopoles emerge from statistically equivalent initial conditions. However, we have found that repeating a certain experiment two or three times (using the same grid configuration) always resulted in a vortex of the same sign, even for different values of Re*. This "bias" in the experiments is most likely introduced just after the forcing, when the grid is lifted out of the fluid by hand. On the other hand, the number of experiments is too low to draw any significant statistical conclusions. It is important to stress that such a bias is not present in the numerical simulations presented in Refs. 3 and 28; approximately half of the runs showing spontaneous spin-up evolves towards a positive monopole and in the other half of the runs a negative monopole is formed.

In order to make a more quantitative comparison between experiments and simulations, one would like to calculate ensemble averages of quantities such as the ratio $\Omega(t) / E(t)$, representing an inverse average size $\lambda$ of the vortices $(\lambda \simeq 2 \pi W \sqrt{E / \Omega})$, and the average number of vortices $V(t)$, as was done for numerical simulations by Clercx et al. ${ }^{11,13}$ However, in most experiments either $\mathrm{Re}^{*}$ or $N$ was varied, and thus no relevant averages could be calculated. Only one small ensemble of three experiments was available for this purpose, in which $N \approx 2.7 \mathrm{rad} \mathrm{s}^{-1}$ and $\mathrm{Re}^{*} \approx 5000$. Figure 12 displays the ensemble averages of $\Omega(t) / E(t)$ [Fig. 12(a)] and $V(t)$ [Fig. 12(b)] as a function of time for these three experiments. Both curves show a power law behavior for several decades, with $\Omega / E \propto t^{-0.5 \pm 0.1}$ and $V \propto t^{-0.5 \pm 0.1}$. Similar power law fits could be made for the individual experiments.

The decay exponents found in Figs. 12(a) and 12(b) do not differ considerably from the values obtained by Hansen et $a l .{ }^{30}$ from laboratory experiments in magnetically forced electrolytes, which are: $\Omega / E \propto t^{-0.47 \pm 0.06}$ and $V \propto t^{-0.70 \pm 0.01}$. Recently, Clercx and Nielsen ${ }^{13}$ performed numerical simulations of decaying 2D turbulence on a square domain with

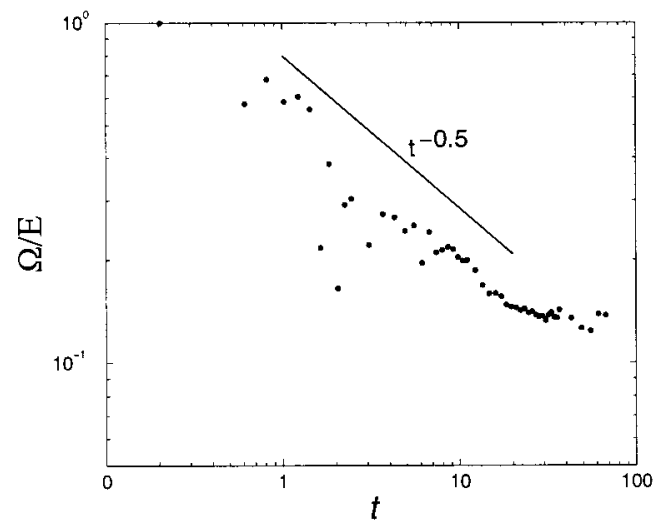

(a)

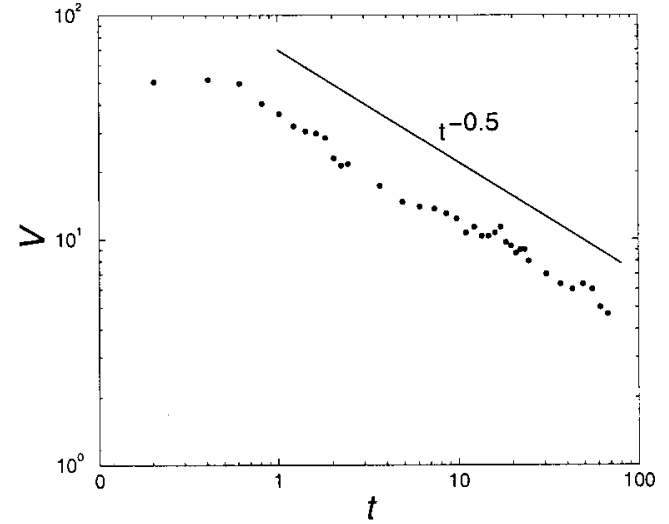

(b)

FIG. 12. Log-log plots of (a) the average ratio $\Omega / E$ and (b) the average number of vortices $V$, both versus the dimensionless time $t$. The data are based on ensemble averages of three experiments with $N \approx 2.7 \mathrm{rad} \mathrm{s}^{-1}$ and $\mathrm{Re}^{*} \approx 5000$ (square container, $L_{0} \approx 0$ ). 


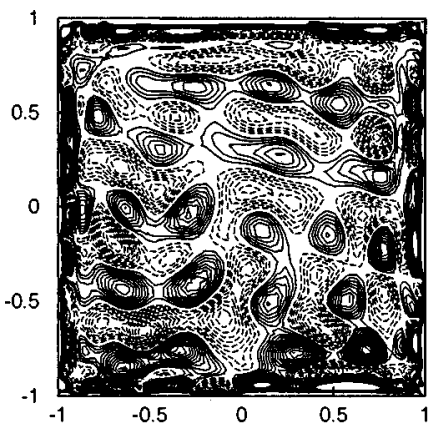

(a) $t=0.5$

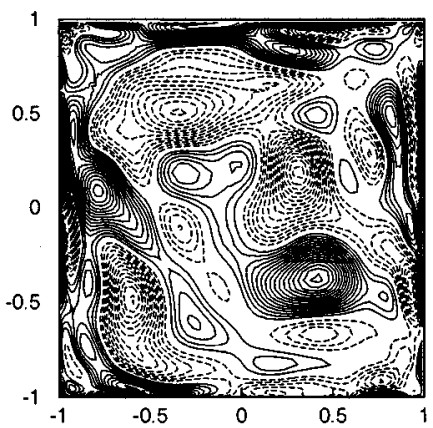

(b) $t=5$

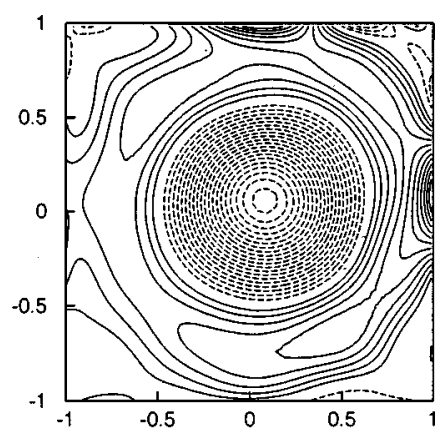

(c) $t=55$

FIG. 13. Vorticity contour plots of an experiment with $\mathrm{Re}^{*}=4000$ and $\left|L_{0}\right|=0.4$ in a square container. Dashed contours represent negative values of vorticity, and solid contours represent positive values. The contour level increment is (a) 2.5, (b) 0.4, and (c) 0.05 .

no-slip boundary conditions, using an initial condition which is close to the experimental situation studied by Hansen et al. ${ }^{30}$ Different Reynolds numbers have been considered: $\mathrm{Re}=1000,2000,5000,10000$, and 20000 . The runs with $\mathrm{Re} \leqslant 2000$ revealed similar scaling behavior as in the experiments by Hansen et al. $.^{30} \Omega / E \propto t^{-0.5}$ and $V \propto t^{-0.7}$. Note that also in our experiments the Reynolds numbers is in the range $500-2000$. The simulations with $\operatorname{Re} \geqslant 5000$ indicated a substantial deviation of the scaling exponents from the experimentally observed values. Only the late-time decay stage, when the kinetic energy of the flow has decreased substantially, it appears that $\Omega / E \propto t^{-0.5}$ and $V \propto t^{-0.7}$.

\section{Experiments with initially nonzero angular momentum}

Although the spontaneous spin-up observed in experiments with $L_{0} \approx 0$ is less pronounced than in numerical simulations with no-slip boundaries, the net angular momentum decreases slowly after the (weak) spin-up has occurred. The question arises how the flow evolves when a net amount of angular momentum is already present in the initial flow. This issue is investigated in six experiments (with buoyancy frequency $N \approx 1.6 \mathrm{rad} \mathrm{s}^{-1}$ ) in which the rods of the grid were arranged in such a way that the initial forcing induced a net angular momentum to the flow (see Table I). Three experi- ments have been performed with $\left|L_{0}\right| \approx 0.4$ and three with $\left|L_{0}\right| \approx 0.6$ [calculated using Eq. (3)], with Reynolds numbers $\mathrm{Re}^{*} \approx 2000, \mathrm{Re}^{*} \approx 3000$ and $\mathrm{Re}^{*} \approx 4000$, respectively. The average decay of the total kinetic energy during these experiments can be fitted with a power law $E \propto t^{\alpha}$, where $\alpha=-0.9$ \pm 0.1 . For the average decay of enstrophy we found $\Omega \propto t^{\beta}$ with $\beta=-1.5 \pm 0.2$. These values for $\alpha$ and $\beta$ are comparable with the average decay exponents found in the experiments with $L_{0} \approx 0$ and $N=1.6 \mathrm{rad} \mathrm{s}^{-1}$ (see Fig. 9).

Figures 13 and 14 show a few snapshots of the vorticity and the stream function, respectively, of an experiment with $\left|L_{0}\right|=0.4$ and $\operatorname{Re}^{*} \approx 4000$ at dimensionless times $t=0.5,5$, and 55. From this experiment it can be concluded that a large-scale vortex starts to dominate the flow at $t \approx 1$. At $t \approx 10$ this vortex forms a triangular-shaped structure (surrounded by three smaller vortices of opposite sign), which rotates in clockwise direction. An almost perfectly circular monopole with a negative core surrounded by a ring of positive vorticity is formed at $t \approx 55$. The scatter plot of $\omega$ versus $\psi$ corresponding to this final monopole is drawn in Fig. 15(a). The peak in this graph corresponds to the interior of the vortex. The sharp $\omega$ - $\psi$-relationship in this region indicates that the monopolar structure is approximately stationary. The (almost) linear shape of the $\omega$ - $\psi$-relation is in agreement with the results reported by Flór ${ }^{16}$ and van de

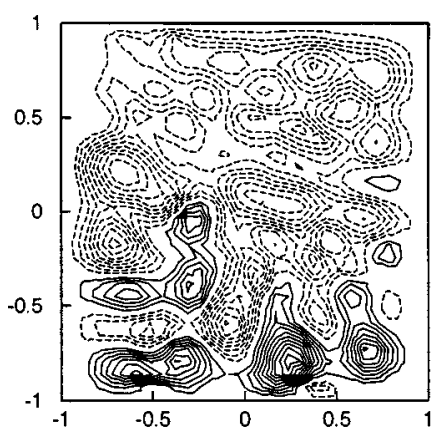

(a) $t=0.5$

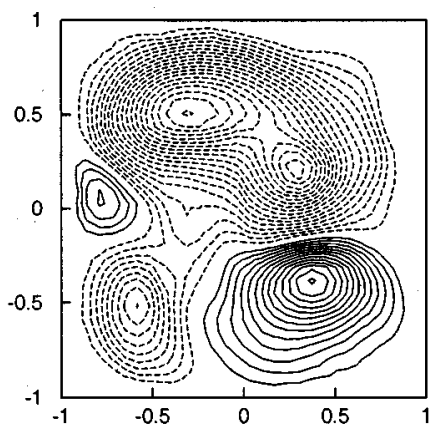

(b) $t=5$

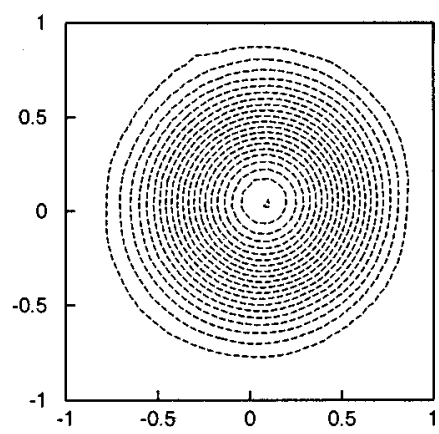

(c) $t=55$

FIG. 14. Stream function contour plots of the same experiment as in Fig. 13 (square container, $\left|L_{0}\right|=0.4$ ). Dashed contours represent negative stream function values, and solid contours represent positive values. The contour level increment is (a) 0.02 , (b) 0.01 , and (c) 0.004 . 


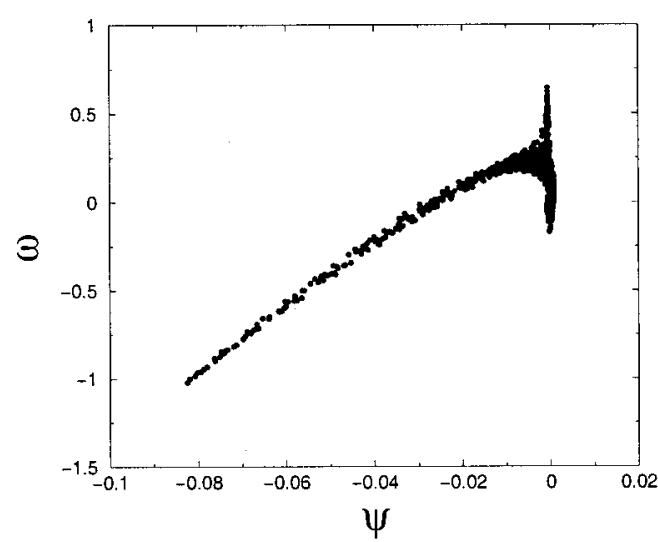

(a)

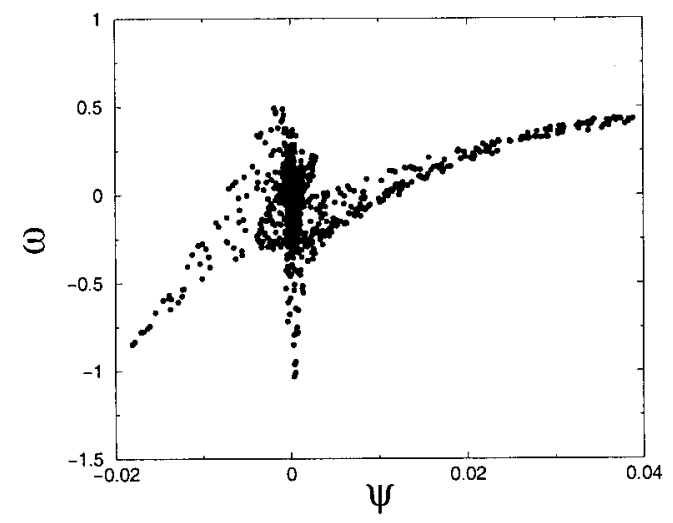

(b)

FIG. 15. Scatter plot of $\omega$ versus $\psi$ corresponding to (a) the monopolar vortex shown in Figs. 13(c) and 14(c) (square container, $\left|L_{0}\right|=0.4$ ), and (b) to the late-time state shown in Fig. $5(\mathrm{f})$ (square container, $L_{0} \approx 0$ ).

Konijnenberg et al.,${ }^{31}$ who observed the appearance of an almost linear $\omega-\psi$-relation in either rake-forced or jet-forced stratified flow in a square container. A similar $\omega$ - $\psi$-profile was observed by Boubnov et $a l^{32}$ in the free decay of a stably stratified flow that was initially forced by an arrangement of sources and sinks around the perimeter of the container.

The $\omega$ - $\psi$-plot of the experiments with $L_{0} \approx 0$ (see contour plots in Figs. 3, 5, and 6) is shown in Fig. 15(b). Note that the $\omega-\psi$ scatter plot is computed after the spontaneous spin-up has occurred $(t \approx 72)$. The difference between the two cases is striking and due to the larger self-organizing capability of flows containing large amounts of angular momentum. The organization into larger vortices occurs on a much shorter time scale when the initial flow field contains a certain amount of angular momentum. This property is nicely illustrated by the normalized net angular momentum depicted in Fig. 11 (solid lines): In the experiments with $L_{0}$ $\neq 0$ a larger value of the normalized angular momentum is reached on a shorter time scale. In Fig. 16(a) the non- normalized dimensionless values of the net angular momentum are plotted versus time for the six experiments with $L_{0}$ $\neq 0$. In this figure the absolute value of the angular momentum decreases strongly during the first few eddy turnover times. One might expect that this fast decay is due to $3 \mathrm{D}$ damping or vertical shearing in the experiments. However, a comparison of these results with the observations from numerical simulations of a purely $2 \mathrm{D}$ flow reveals that this hypothesis does not hold. In Fig. 16(b) the net angular momentum is plotted versus time for a set of numerical simulations with $\mathrm{Re}=1000$. The initial conditions of these simulations are constructed according to the procedure described in Ref. 11, but the random coefficients of the initial velocity field are chosen in such a way that the flow contains a net nonzero angular momentum. By comparing Figs. 16(a) and 16(b) we see that the fast decrease of angular momentum in the initial few eddy turnover times of the simulations is of the same order as in the experiments. This means that this effect is probably due to strong dissipation in the boundary layers, which slows down the net large-scale rotation of the

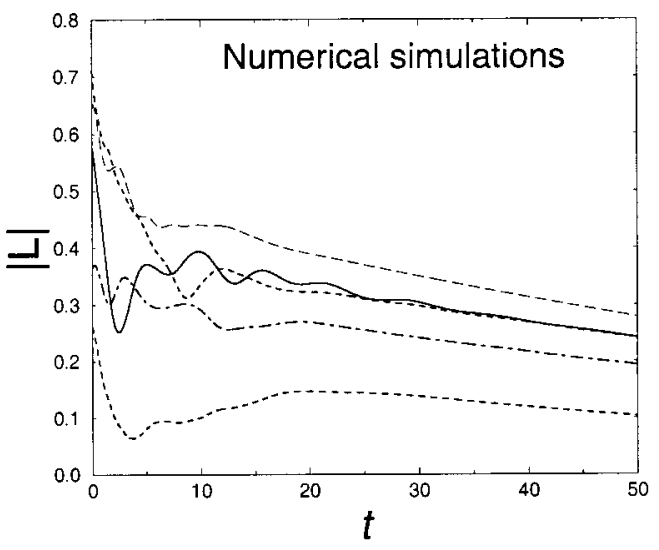

(b)

FIG. 16. Dimensionless net angular momentum versus dimensionless time for (a) six experiments in a square container with $L_{0} \neq 0$ and (b) five numerical runs on a square domain with no-slip boundaries, $\operatorname{Re}=1000$ and $L_{0} \neq 0$. 


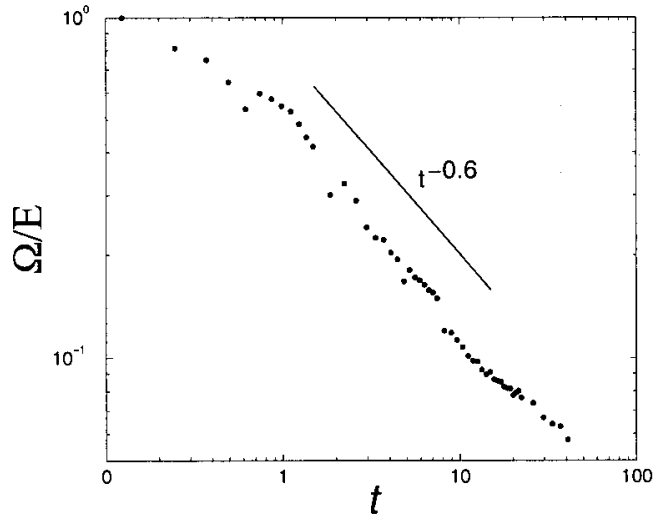

(a)

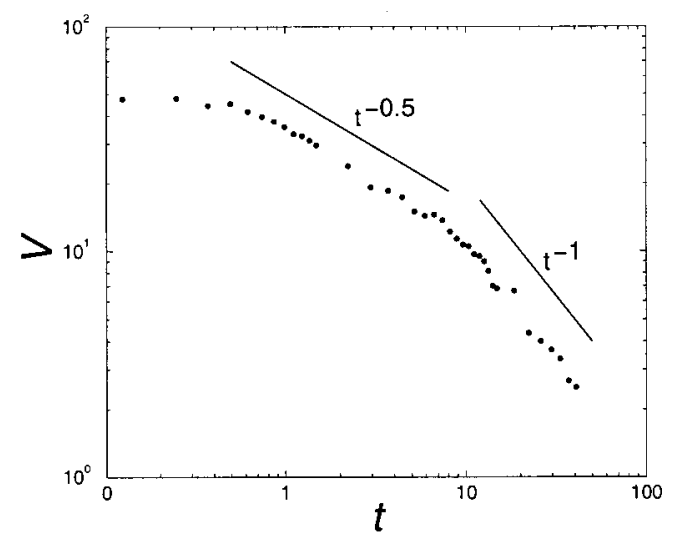

(b)

FIG. 17. Log-log plots of (a) the average ratio $\Omega / E$ and (b) the average number of vortices $V$, both versus the dimensionless time $t$. The data are based on ensemble averages of six experiments in a square container with $L_{0} \neq 0$.

flow. After this transient stage, the angular momentum in the simulations decays more slowly than in the experiments, as can be inferred from Figs. 16(a) and 16(b). This difference is most likely due to the strong vertical shearing in the experiments, as discussed in Sec. III B.

It was found that the decay properties such as the number of vortices $V(t)$ and the ratio $\Omega(t) / E(t)$ satisfy approximately the same power laws for all experiments with $L_{0}$ $\neq 0$, although the values of $\mathrm{Re}^{*}$ and $L_{0}$ were different for each experiment. Nevertheless, some indications are present supporting the conjecture that runs with $L_{0}>0$ yield a somewhat faster self-organization of the decaying turbulent flow. Figure 17 shows a log-log plot of the average ratio $\Omega(t) / E(t)$ [see Fig. 17(a)] and the average number of vortices $V(t)$ [see Fig. 17(b)] for the six experiments with nonzero $L_{0}$. The curve in Fig. 17(a) can be fitted with a power law $\Omega / E \propto t^{-0.6 \pm 0.1}$. This power law is comparable (although with a slightly larger exponent) with the power law for $\Omega / E$ obtained from experiments with $L_{0} \approx 0$ (cf. Fig. 12). However, one should be very careful with this comparison, since these experiments with $L_{0} \approx 0$ were performed in a much stronger stratification. Figure 17(b) contains a log-log plot of the averaged number of vortices. Two ranges with different power laws are found in this case: $V \propto t^{-0.5 \pm 0.1}$ for $t$ $\lesssim 10$ and $V \propto t^{-1.0 \pm 0.1}$ for $t \gtrsim 10$. The value for the first stage $(t \lesssim 10)$ is similar to the power law found for experiments with $L_{0} \approx 0$ (Fig. 12). Apparently, the net large-scale rotation of the system of small vortices which is observed during this stage (Figs. 13 and 14) does not considerably influence the decay properties of vortex statistics in these experiments. The steep decrease of $V$ at $t \gtrsim 10$ reflects the faster organization of flows with finite $L_{0}$ compared with the experiments in which $L_{0} \approx 0$.

Combining the experimental and numerical results with $L_{0} \approx 0$ and $L_{0} \neq 0$ it might be tempting to draw the following conclusion. A particular optimal value $L_{0, \text { opt }}$ of the initial angular momentum exists that suppresses enhanced decay of $L$ (observed when $L_{0} \gtrsim L_{0 \text {,opt }}$ ) and prevents spontaneous spin-up of the flow (observed when $L_{0} \lesssim L_{0 \text {,opt }}$ ). It might, therefore, be expected that the self-organization process is most efficient for $L_{0} \sim L_{0 \text {,opt }}$, because then a balance exists between production and destruction of angular momentum. The flow consists then of a shielded rotating vortex in the interior of the domain without producing too many smallscale vortices in the boundary layers.

\section{LABORATORY EXPERIMENTS IN A CIRCULAR CONTAINER}

A second series of experiments has been performed in a circular container with a diameter of $92 \mathrm{~cm}$. The Reynolds number of this quasi-2D flow is defined as $\mathrm{Re}^{*}=R U / \nu$, with $R$ the radius of the container, $U$ the root-mean-squared velocity of the initial flow field, and $\nu$ the kinematic viscosity of the fluid. Using similar arguments as in Sec. III the error in the estimated value of $\mathrm{Re}^{*}$ is of the order of $15 \%$.

In the present experiments, $\mathrm{Re}^{*}$ is approximately 5000 (the actually measured value differs in each experiment), but the effective Reynolds number related to the measured quasi-2D flow is much smaller than $\mathrm{Re}^{*}$ (see Sec. III A). In the experiments performed in a circular container, the energy decays approximately $80 \%$ within 30 eddy turnover times. This decay rate is of the same order as the decay rate of kinetic energy observed in the numerical simulations of LMJ. Therefore, we assume that the "effective" Reynolds number of the laboratory experiments described in this section is comparable with the Reynolds numbers used in the numerical simulations by LMJ (which vary from 700 to 1250).

Throughout this section, all variables are made dimensionless using the length scale $R$ and the eddy-turnover time $t=R / U$. Using polar coordinates $\mathbf{r}=(r, \theta)$, the net angular momentum $L$, defined with respect to the center of the domain, and the total kinetic energy $E$ are defined as

$$
\begin{aligned}
& L=\int_{0}^{1} \int_{0}^{2 \pi} \hat{\mathbf{k}} \cdot(\mathbf{r} \times \mathbf{v}) r d r d \theta, \\
& E=\frac{1}{2} \int_{0}^{1} \int_{0}^{2 \pi}|\mathbf{v}|^{2} r d r d \theta .
\end{aligned}
$$


TABLE II. Characteristic parameters of the experiments performed in a circular container. $\mathrm{Re}^{*}=$ Reynolds number, $L_{0}=$ initial net angular momentum, $N$ =buoyancy frequency. The grid configurations (fourth column) are specified in the Appendix.

\begin{tabular}{cclllc}
\hline \hline $\begin{array}{c}\text { Number of } \\
\text { experiments }\end{array}$ & $\mathrm{Re}^{*}$ & $\left|L_{0}\right|$ & Grid & Stratification & $N\left(\mathrm{rad} \mathrm{s}^{-1}\right)$ \\
\hline 1 & 5000 & 0 & $\mathrm{C} 1$ & two-layer & 2.7 \\
2 & 5000 & 0 & $\mathrm{C} 2$ & two-layer & 2.7 \\
1 & 4000 & 0 & $\mathrm{C} 2$ & linear & 2.7 \\
1 & 4000 & 0.20 & $\mathrm{C} 3$ & two-layer & 2.7 \\
1 & 4000 & 0.37 & $\mathrm{C} 4$ & two-layer & 2.7 \\
1 & 4000 & 0.35 & $\mathrm{C} 4$ & linear & 2.7 \\
\hline \hline
\end{tabular}

It is worthwhile to emphasize that, on a circular domain, both $L$ and $E$ are exactly conserved in the case of vanishing viscosity. Conservation of energy is easily understood by realizing that $d E / d t=-2 \nu \Omega \rightarrow 0$ for vanishing viscosity (assuming that $\Omega \propto \nu^{-\gamma}$ with $\gamma<1$ ). The conservation of $L$ can be verified by putting $1 / \operatorname{Re}=0$ and $\mathbf{r} \cdot d \mathbf{s}=0$ in Eq. (6). The condition $\oint_{\partial \mathcal{D}} p \mathbf{r} \cdot d \mathbf{s}=0$ corresponds to the fact that normal stresses acting on the fluid at the circular boundary do not induce a torque relative to the center of the domain. In the presence of viscosity, the decay rate of $L$ on a circular domain with radius 1 and no-slip boundaries can be expressed as

$$
\frac{d L}{d t}=\frac{1}{\operatorname{Re}} \int_{0}^{2 \pi} \omega(1, \theta) d \theta,
$$

with $\omega(1, \theta)$ the vorticity at the boundary of the container.

\section{A. Spatial flow evolution}

Several experiments have been performed in a circular container with different values of $\mathrm{Re}^{*}$ and $L_{0}$. Some characteristic parameters of the performed experiments are listed in Table II. Figure 18 shows contour plots of the stream function of a typical experiment with approximately zero initial net angular momentum $\left(L_{0} \approx 0\right)$ at dimensionless times $t=1,5,10,20,40$, and 80, respectively. Figure $18(\mathrm{a})$ is taken soon after the forcing was stopped. At this stage the small vortices, which are formed in the wake of the rods, have already started to evolve according to the usual behavior of 2D turbulence: like-signed vortices merge, oppositely signed vortices form dipoles, and all vortices grow in size. At $t \approx 1$ less than ten vortices of different strength and size are left.

The two strong vortices in the right part of the container [Fig. 18(a)] form a dipolar structure at $t \approx 5$ [Fig. 18(b)], which moves to the left side of the container, where it pushes against the wall [Figs. 18(c) and 18(d)]. Owing to the no-slip boundary condition, thin boundary layers are formed, in which the shear flow has oppositely signed vorticity. Subsequently, these filamentary vorticity patches are advected away from the boundary, into the interior. As a result, two new vortices are gradually being formed just behind the large dipole [Fig. 18(d)]. Together with the "old" dipole, these new vortices form an asymmetric, quadrupolar structure [Fig. 18(e)]. The two vortices in the lower left part of the container increase in size and strength, until they form a new, domain-filling dipole at $t \approx 80$ [Fig. 18(f)].

The experiment shown in Fig. 18 has been repeated several times. All experiments show a tendency to form dipolar structures, which move to the wall, push against it, and form two new vortices at the rear, but the particular time evolution is different in each experiment. Since dipoles and asymmetric quadrupoles are formed alternatingly, the flow does not reach a clear "final" quasi-stationary state. This observation is confirmed by the vorticity fields plotted in Figs. 19(a) and 19(b) for the same experiment as shown in Fig. 18 at $t=40$ and $t=80$, respectively. The corresponding scatter plots of $\omega$ versus $\psi$ are plotted in Fig. 20. Unfortunately, the experimental scatter in the vorticity is quite large, and it was not pos-

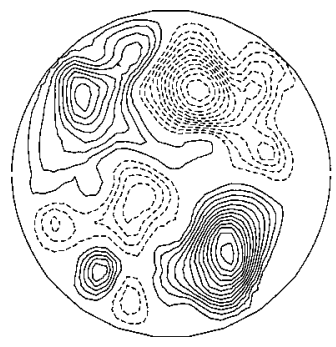

(a) $t=1$

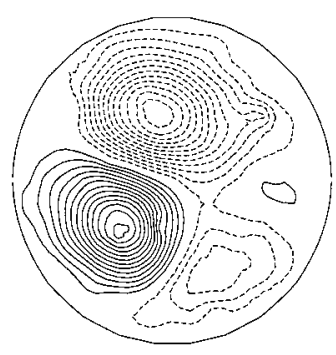

(d) $t=20$

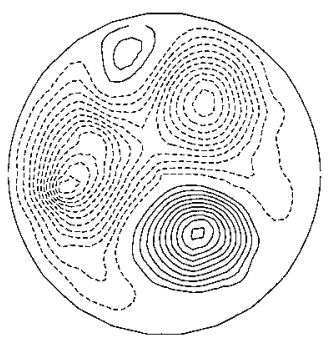

(b) $t=5$

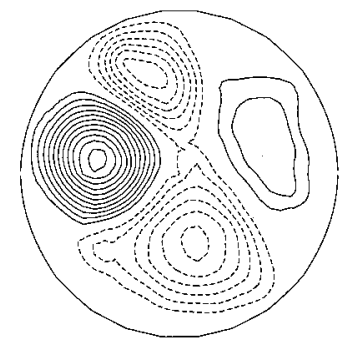

(e) $t=40$

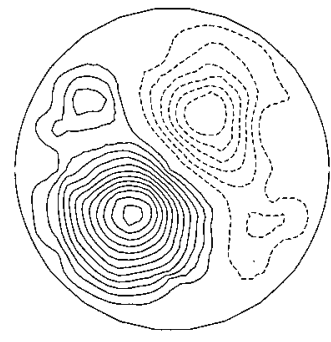

(c) $t=10$

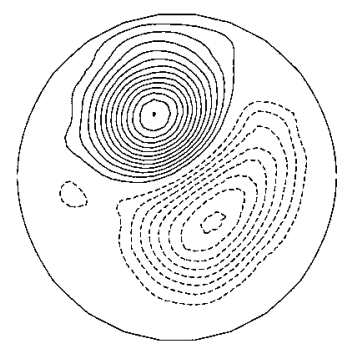

(f) $t=80$
FIG. 18. Stream function contour plots of an experiment with $L_{0} \approx 0$ in a circular container. Dashed contours represent negative stream function values, and solid contours represent positive values. The contour level increment is (a) 0.02 , (b)-(d) 0.01 , (e) 0.008 , and (f) 0.004 


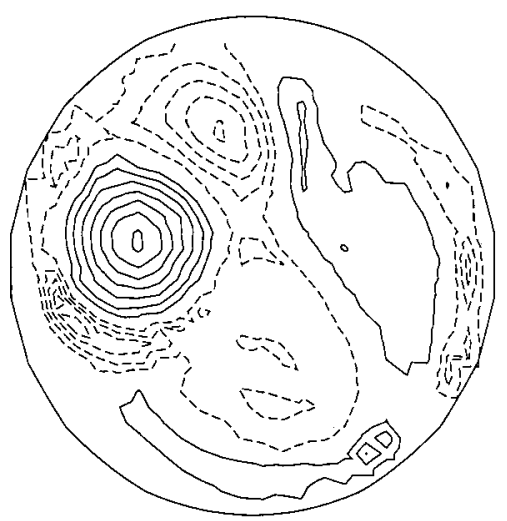

(a) $t=40$

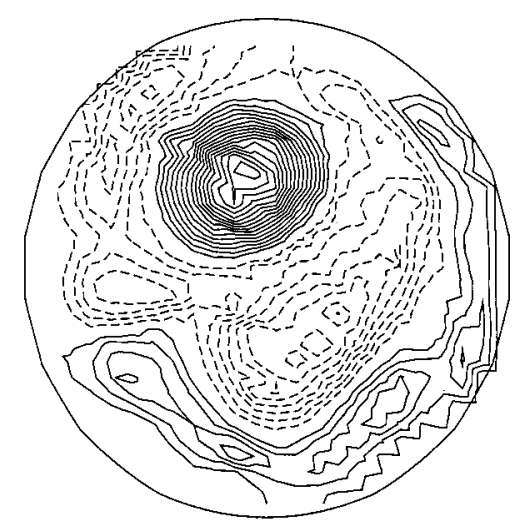

(b) $t=80$
FIG. 19. Vorticity contour plots of the late-time states emerging in the experiment shown in Fig. 18 (circular container, $L_{0} \approx 0$ ). Dashed contours represent negative values of vorticity, and solid contours represent positive values. The contour level increment is (a) 0.5 and (b) 0.1 . sible to obtain clear vorticity plots for the experiments in a circular container at earlier stages of the flow evolution (the data fitting scheme described in Sec. III is based on a Chebyshev expansion in two directions and is therefore not applicable to a circular domain). In spite of the experimental errors, the shape of the $\omega$ - $\psi$-plots drawn in Figs. 20(a) and 20(b) suggests that there is no functional relationship between $\omega$ and $\psi$. The vorticity plots presented in Fig. 19 show a striking similarity with the late-time vorticity plots obtained by LMJ from numerical simulations of decaying 2D turbulence in a circular container with no-slip boundary conditions. As an example, Fig. 21 shows the vorticity plots obtained from a simulation by Li et al. ${ }^{15}$ with $\mathrm{Re}=707$, after $t=30$ and $t=100$ eddy turnover times, respectively. The $\omega-\psi$-plot corresponding to the field in Fig. 21(a) is plotted in Fig. 22. We remark that the outer branches of the $\omega$ - $\psi$-plots drawn in Figs. 20 and 22 show a tendency to become linear.

The decay of kinetic energy obtained from the experiment shown in Fig. 18 is plotted in Fig. 23 (filled symbols). This picture suggests the existence of two regimes in which the energy decays according to a power law: Fitting the experimental data with the curve $E \propto t^{\alpha}$, we find $\alpha=-0.5 \pm 0.1$ for $t \lesssim 10$ and $\alpha=-1.4 \pm 0.2$ for $t \gtrsim 10$. The kinetic energy of 3D turbulent motions in the first few seconds of decaying stratified turbulence is partly converted into potential energy associated with internal waves. At later times, this potential energy may partly be converted back into kinetic energy. This latter mechanism could explain the temporarily decreased dissipation rate at $t \lesssim 10$. However, one should be careful with this conclusion, since this phenomenon was not observed clearly in the experiments on decaying stratified turbulence in a square container and comparable values of $N(N \approx 2.7)$ (cf. Fig. 9, open symbols). For $t \gtrsim 10$ the dissipation of kinetic energy is dominated by vertical shear, ${ }^{17,18}$ and the power-law behavior is similar as in the square-container experiment.

In order to investigate the influence of the unstratified layers above and beneath the interface in a two-layer stratification, the experiment described above was repeated in a linear stratification. For this purpose the container was filled slowly from beneath using the so-called two-bucket method, ${ }^{23}$ thereby creating a linear increase of the density over a depth of $20 \mathrm{~cm}$. The gradient of the stratification was chosen such that the buoyancy frequency $N$ matched the buoyancy frequency at the interface of the two-layer experiments described above $\left(N \approx 2.7 \mathrm{rad} \mathrm{s}^{-1}\right)$. The energy decay of this experiment, plotted in Fig. 23 (open symbols), is not significantly different from the decay observed in a two-layer

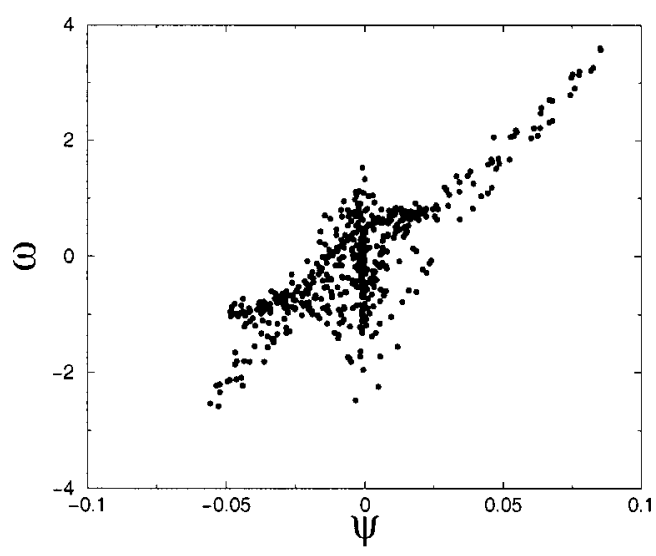

(a) $t=40$

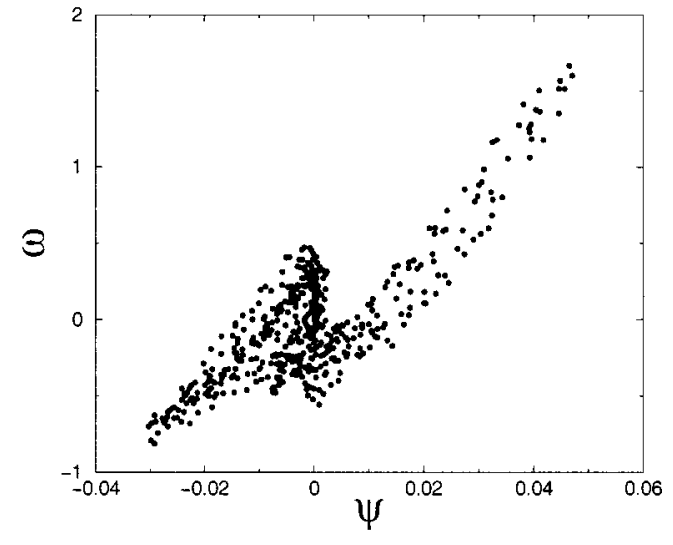

(b) $t=80$

FIG. 20. Scatter plot of $\omega$ versus $\psi$ corresponding to the vorticity plots shown in Fig. 19 (circular container, $L_{0} \approx 0$ ). 


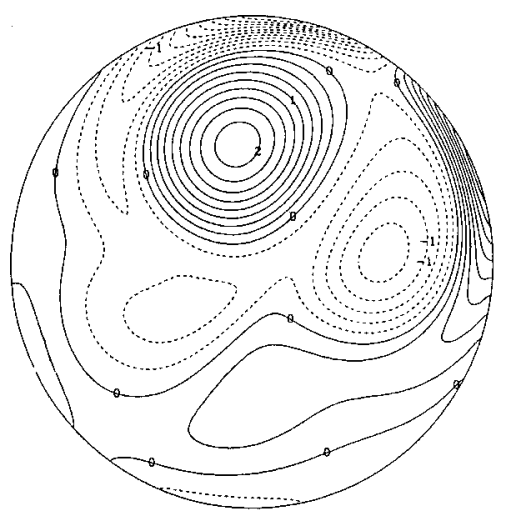

(a) $t=30$

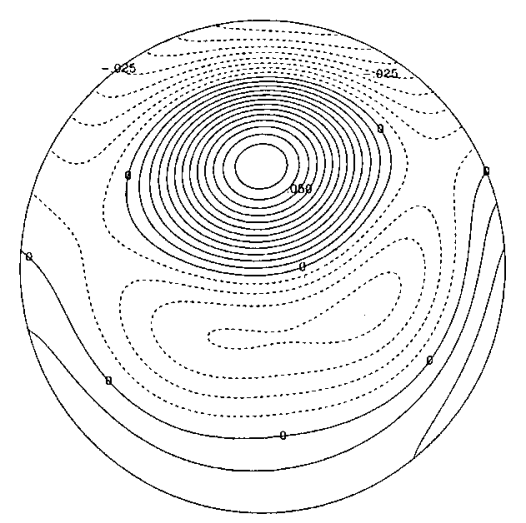

(b) $t=100$
FIG. 21. Vorticity contour plots of the late-time states emerging in a numerical simulation of decaying $2 \mathrm{D}$ turbulence on a circular domain with no-slip boundary conditions, $L_{0} \approx 0$ and $\mathrm{Re}=707$, performed by Li et al. (1997). Dashed contours represent negative values of vorticity, and solid contours represent positive values. stratification (same figures, filled symbols). A similar comparison was made between two experiments with an initially nonzero amount of angular momentum (see Sec. IV B). Apparently, the linearly stratified interface in the two-layer fluid is thick enough to shield the quasi-2D motions in the center of this interfacial layer from the (almost motionless) unstratified layers above and beneath the interface.

\section{B. Conservation of angular momentum?}

The spatial flow evolution appears to be quite different when the initial flow contains a finite (nonzero) amount of net angular momentum. Figure 24 shows contour plots of the stream function of an experiment in which the forcing rods were arranged in the grid in such a way that they induced a net angular momentum $\left|L_{0}\right|=0.37$. For comparison: A solidbody rotation of the same amount of fluid, with the same kinetic energy $\left(E_{0}=1.57\right)$, would have an angular momentum $\left|L_{s b}\right|=2.22$. The initial flow again contains a more or less homogeneous distribution of small-scale vortices, which organize into two large structures at $t \approx 5$. However, in contrast to the evolution in the previous experiment (Fig. 18), the initial flow field contains a net large-scale rotation super-

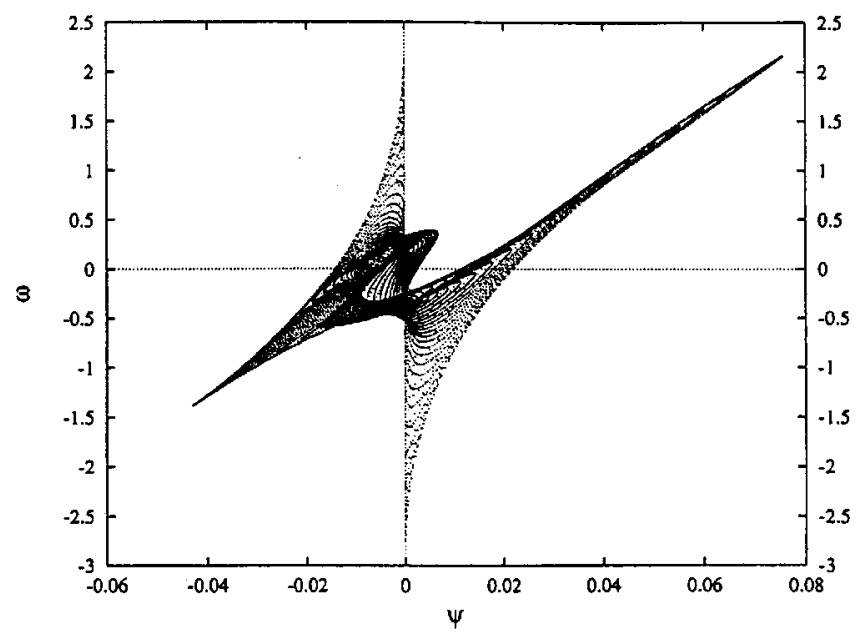

FIG. 22. Scatter plot of $\omega$ versus $\psi$ corresponding to the vorticity plot shown in Fig. 21(a), obtained from a numerical simulation of decaying 2D turbulence on a circular domain with no-slip boundary conditions, $L_{0} \approx 0$ and $\mathrm{Re}=707$ performed by Li et al. (1997). imposed on the small-scale vortices, which is clearly visible in Fig. 24(a). This large-scale rotation dominates the flow evolution [Figs. 24(a)-24(d)] and finally, at $t \approx 60$, one large monopolar vortex fills the domain completely. A similar configuration was found in all experiments with a net nonzero angular momentum in the initial flow.

In Fig. 25(a) some contours of the vorticity field are plotted for the quasi-stationary final state (a monopolar vortex) from the sequence shown in Fig. 24. The corresponding scatter plot of $\omega$ versus $\psi$ is drawn in Fig. 25(b). In this plot, the leftmost points correspond to the region closest to the center of the vortex, whereas the right and upper points correspond to regions near the wall. Although the flow in this experiment has attained a clear quasi-stationary state, the $\omega$ - $\psi$-plot still contains a large amount of scatter, which is most probably due to a combination of experimental errors and viscous effects. Nevertheless, the vorticity and $\omega$ - $\psi$-plots shown in Fig. 25 show a good agreement with the late-time monopolar vortices emerging in the numerical simulations with finite $L_{0}$ by LMJ. Furthermore, a similar configuration was observed by Flór ${ }^{16}$ in experiments on decaying grid-

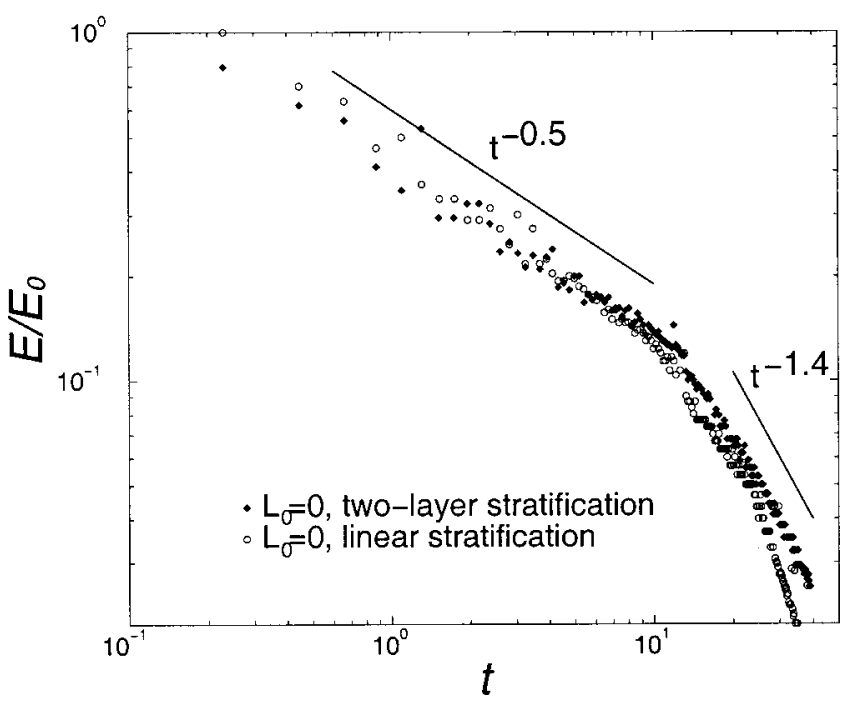

FIG. 23. Normalized kinetic energy $E / E_{0}$ for the experiment shown in Fig. 18 (circular container, $L_{0} \approx 0$ ) performed in a two-layer stratification (filled symbols) and for a similar experiment performed in a linear stratification (open symbols). 


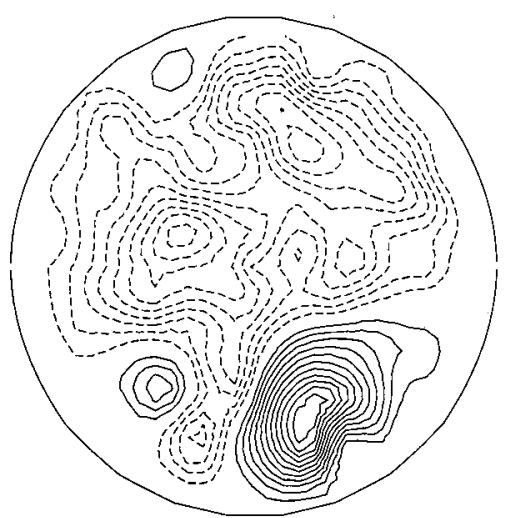

(a) $t=1$

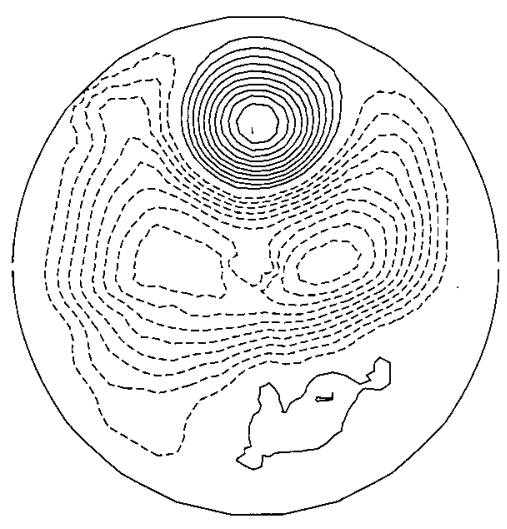

(c) $t=15$

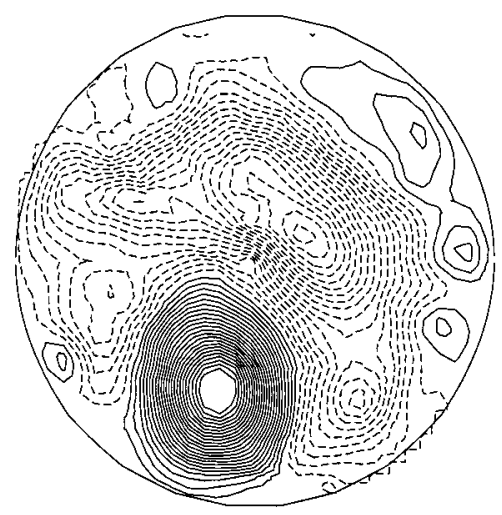

(b) $t=5$

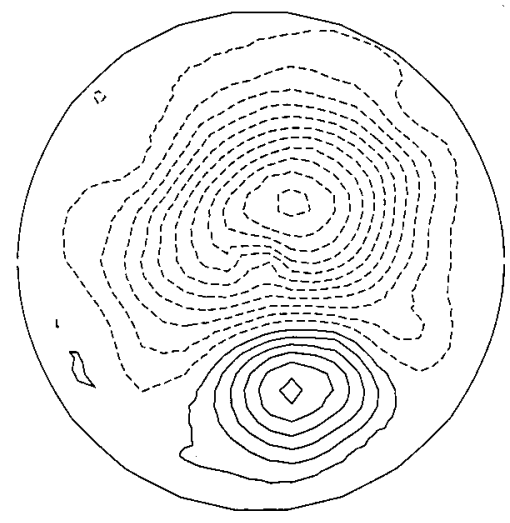

(d) $t=30$
FIG. 24. Stream function contour plots of an experiment with $\left|L_{0}\right|=0.37$ in a circular container. Dashed contours represent negative stream function values, and solid contours represent positive values. The contour level increment is (a) and (b) 0.02 , (c) and (d) 0.01 . generated turbulence in a stratified flow inside a circular container. In these experiments the initial angular momentum could not be controlled, since the forcing was performed manually. The fact that Flor observed the formation of a stationary monopole in all experiments in a circular con- tainer indicates that the manual forcing in these experiments most probably introduced a nonzero net angular momentum to the flow.

The role of the net angular momentum becomes even more clear in Fig. 26, which shows the evolution of the net

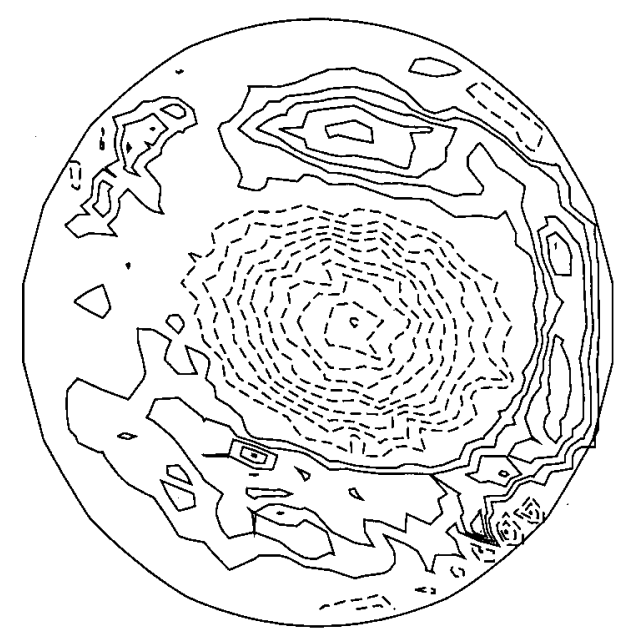

(a)

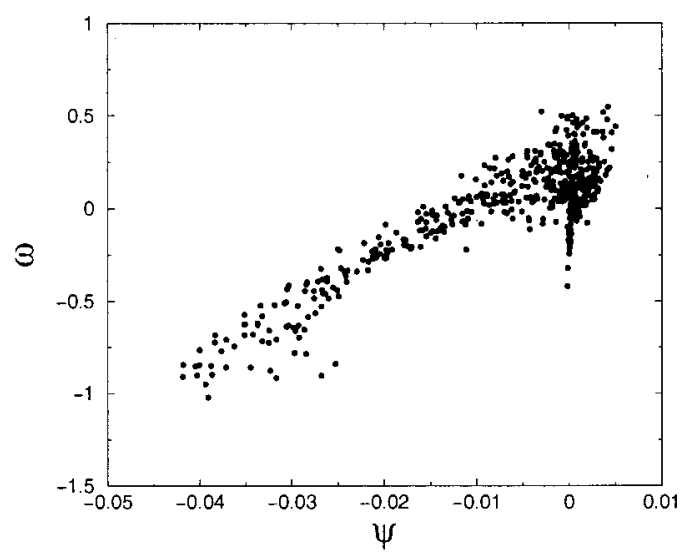

(b)

FIG. 25. (a) Contours of vorticity and (b) scatter plot of $\omega$ versus $\psi$, both corresponding to the late-time state of the experiment shown in Fig. 24 (circular container, $\left.\left|L_{0}\right|=0.37\right)$ at $t=60$. In (a) dashed contours represent negative values of vorticity, and solid contours represent positive values. The contour level increment is 0.1 . 


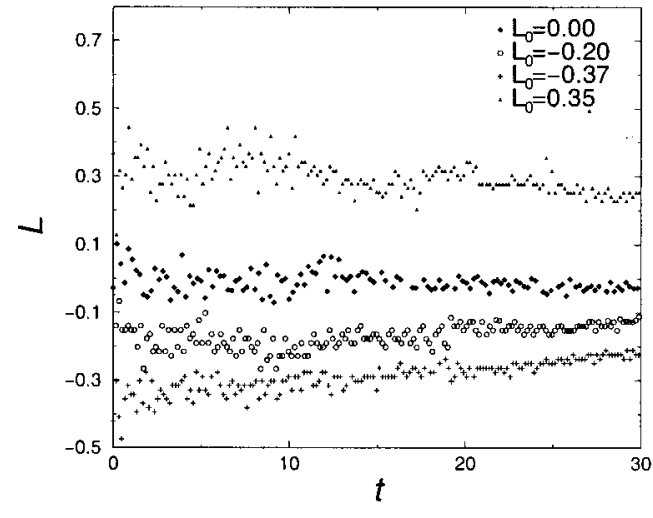

(a)

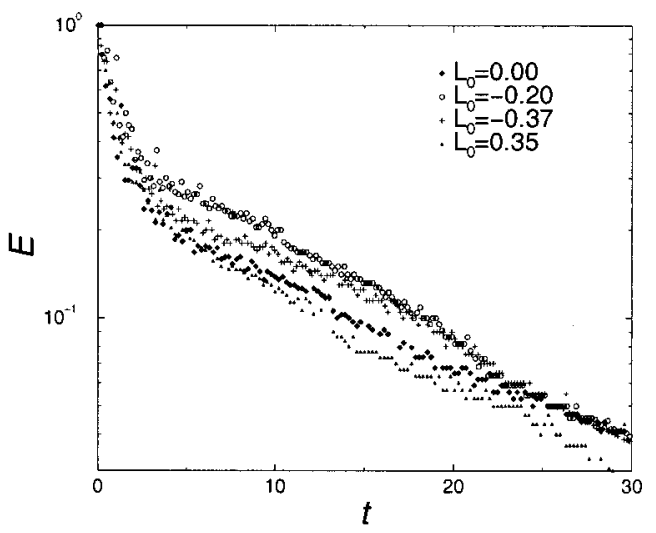

(b)

FIG. 26. (a) The dimensionless net angular momentum plotted versus dimensionless time and (b) the normalized kinetic energy plotted logarithmically versus dimensionless time, both for four different experiments in a circular container: $L_{0}=0, L_{0}=-0.20, L_{0}=-0.37$, and $L_{0}=0.35$.

angular momentum [Fig. 26(a)] and the total kinetic energy [Fig. 26(b)] during the first 30 eddy-turnover times of four experiments: One experiment with $L_{0} \approx 0$ (that is, the experiment shown in Fig. 18) and three experiments with $L_{0} \neq 0$ $\left(L_{0}=-0.20, L_{0}=-0.37\right.$ and $L_{0}=0.35$, respectively). The data points with $L_{0}=-0.37$ correspond to the experiment shown in Fig. 24. (The experiment with $L_{0}=0.35$ was performed in a linear stratification with $N \approx 2.7 \mathrm{rad} \mathrm{s}^{-1}$; the other three are performed in a two-layer fluid with $N$ $\approx 2.7 \mathrm{rad} \mathrm{s}^{-1}$ in the interfacial layer.) These plots clearly show that, apart from some fluctuations during the first 15 eddy turnover times, the average magnitude of the angular momentum decays much slower than the kinetic energy. The fluctuations in the angular momentum during the first 15 eddy turnover times are due to rapidly changing configurations of small-scale vortices, which are also observed in numerical simulations with no-slip boundaries on circular or square domains [cf. Fig. 7(b)]. Since the net angular momentum is not a rigorous constant of the motion in the presence of viscosity [cf. Eq. (12)], the slow decay of angular momentum is one of the most surprising results of experiments and simulations of decaying (quasi-)2D turbulence on a circular domain with no-slip boundaries.

\section{CONCLUSIONS}

The self-organization of confined two-dimensional flows depends strongly on the geometry of the domain and on the type of boundary condition. This conclusion is founded on numerical simulations ${ }^{11}$ and the laboratory experiments of decaying (quasi-)2D turbulence in different geometries as described in this paper. Laboratory experiments of decaying stratified turbulence in square and circular containers confirm the results of numerical simulations with no-slip boundary conditions, in spite of the strong vertical shearing and the three-dimensional structure of the flow in the experiments. This observation implies that purely $2 \mathrm{D}$ numerical simulations provide a useful tool to investigate the self-organization of quasi-2D stratified flows in more detail.

The laboratory experiments in stably stratified fluids have revealed some fundamental differences in the decay scenario of quasi-2D turbulence in square and circular containers. In the square container, weak spin-up of the flow is observed in experiments in a weakly stratified fluid (with buoyancy frequency $N \approx 1.6 \mathrm{rad} \mathrm{s}^{-1}$ ), in which no net angular momentum was induced to the initial flow. This effect is observed to be more pronounced in numerical simulations with higher Reynolds numbers $(\operatorname{Re}>2000)$, but these values of Re could not be obtained in the laboratory experiments due to strong vertical shearing between the different layers of the fluid, and due to the generation of internal waves. In experiments in which a finite amount of net angular momentum was introduced to the initial flow, the organization into a quasi-stationary monopolar structure occurs on a shorter time scale than in the experiments with zero initial angular momentum. The net angular momentum in these experiments decreases strongly during an initial transient stage of about five dimensionless time units. Thereafter, the angular momentum decays much more slowly than the total kinetic energy. These observations are in agreement with the results of numerical simulations in containers with no-slip boundaries, with $\mathrm{Re}=1000$ and a finite amount of net angular momentum in the initial condition. In the circular case, the net angular momentum contained in the initial flow field appears to be a better conserved global quantity than the total kinetic energy, and its absolute value has a remarkable influence on the evolution towards the "final" quasi-stationary state. When the initial flow contains a finite (nonzero) amount of angular momentum, the flow organizes rapidly towards a large domain-filling monopole. When no net angular momentum is contained in the initial flow, no clear quasi-stationary state emerges. Instead, dipolar and quadrupolar structures are being formed alternately. These results confirm the conclusions by $\mathrm{LMJ}^{1,14,15}$ concerning the numerically obtained decay scenario of 2D turbulence in a circular container with no-slip boundaries.

An issue not discussed yet is an important difference in the decay scenarios of decaying 2D turbulence in square and in circular containers with $L_{0}=0$. For square containers spontaneous spin-up has been observed and no spin-up occurs in the case of a circular container. The main reason is 
the following: By (numerically) analyzing the time rate of change of $L$ [see Eq. (6)] it is found that the normal stress contribution $1 / \rho \oint_{\partial \mathcal{D}} p \mathbf{r} \cdot d \mathbf{s}$ is much larger than the shear stress term $1 / \operatorname{Re} \oint_{\partial \mathcal{D}} \omega(\mathbf{r} \cdot \hat{\mathbf{n}}) d s$, and spontaneous spin-up is fully determined by the normal stress contribution. This contribution is, however, absent in the circular case $(\mathbf{r} \cdot d \mathbf{s}=0)$ and $d L / d t$ is determined by the much weaker shear stress contribution which does not promote spontaneous spin-up of the flow.

Finally, we would like to stress that the experimental observations confirm, at least in a qualitative sense, the results obtained by numerical simulations of decaying 2D turbulence with no-slip boundaries in both circular and square domains. This conclusion is not obvious, since in literature the results of laboratory experiments have often been compared with numerical simulations or theoretical approaches in which different boundary conditions (stress-free or doubly-periodic) were used. Furthermore, the qualitative agreement between laboratory experiments and 2D simulations indicates that large-scale processes in stably stratified fluids are indeed in good approximation 2D for the range of Reynolds numbers considered in the experiments, although the dissipation of kinetic energy is much stronger in the experiments due to vertical shear.

\section{ACKNOWLEDGMENTS}

One of the authors (S.R.M.) is supported by the Netherlands Geoscience Foundation (GOA) with financial aid from the Netherlands Organization for Scientific Research (NWO).

\section{APPENDIX: GRID CONFIGURATIONS}

In this appendix the grid configurations used in the laboratory experiments are specified by means of the $y$-coordinates of the rods (in $\mathrm{cm}$ ), defined in Fig. 2. The net angular momentum $L_{0}$ induced on the flow can be calculated from these $y$-coordinates by using Eq. (3).

(i) Square container

Grid S1: $( \pm 40, \pm 38, \pm 36, \pm 34, \pm 32, \pm 22, \pm 20, \pm 18$, $\pm 16, \pm 14, \pm 4, \pm 2,0)$.

Grid S2: $( \pm 48, \pm 46, \pm 44, \pm 42,34, \pm 32, \pm 30, \pm 28$, $-22, \pm 20,-19,18,-17,16,14,-12,-10$, $-8, \pm 6,4,2,0)$.

Grid S3: $( \pm 40,38, \pm 36,34, \pm 32, \pm 22,20, \pm 18,16$, $\pm 14, \pm 4,2)$.

Grid S4: $( \pm 40,38,36,34,32,-31, \pm 22,20,18,16,14$, $-13, \pm 4,2)$.

(ii) Circular container

Grid C1: $( \pm 33, \pm 31, \pm 29, \pm 27, \pm 25, \pm 17, \pm 15, \pm 13$, $\pm 11, \pm 3, \pm 1)$.

Grid C2: $(-33, \pm 31, \pm 29, \pm 27,21, \pm 19,18,-17,16$, $-15,-13,11,9,7, \pm 5,-3,-1)$.

Grid C3: $(-32, \pm 30, \pm 28,26,-23,-21,-19,18$, $-17,16,14,-12,-10,-8, \pm 6,4,2)$.

Grid C4: $(-32, \pm 30,-28,26,-23,-21,-19,18$, $-17,14,-12,-10,-8, \pm 6,2)$.
${ }^{1} \mathrm{~S}$. Li and D. Montgomery, "Decaying two-dimensional turbulence with rigid walls," Phys. Lett. A 218, 281 (1996).

${ }^{2}$ W. H. Matthaeus, W. T. Stribling, D. Martinez, S. Oughton, and D. Montgomery, "Decaying, two-dimensional, Navier-Stokes turbulence at very long times," Physica D 51, 531 (1991).

${ }^{3}$ H. J. H. Clercx, S. R. Maassen, and G. J. F. van Heijst, "Spontaneous spin-up during the decay of 2D turbulence in a square container with rigid boundaries," Phys. Rev. Lett. 80, 5129 (1998).

${ }^{4}$ S. R. Maassen, H. J. H. Clercx, and G. J. F. van Heijst, "Decaying quasi-2D turbulence in a stratified fluid with circular boundaries," Europhys. Lett. 46, 339 (1999).

${ }^{5}$ W. H. Matthaeus and D. Montgomery, "Selective decay hypothesis at high mechanical and magnetic Reynolds numbers," Ann. (N.Y.) Acad. Sci. 357, 203 (1980)

${ }^{6} \mathrm{~J}$. C. McWilliams, "The emergence of isolated coherent vortices in turbulent flows," J. Fluid Mech. 146, 21 (1984).

${ }^{7}$ J. C. McWilliams, "The vortices of two-dimensional turbulence," J. Fluid Mech. 219, 361 (1990).

${ }^{8}$ P. Santangelo, R. Benzi, and B. Legras, "The generation of vortices in high-resolution, two-dimensional, decaying turbulence and the influence of initial conditions on the breaking of self-similarity," Phys. Fluids A 1, 1027 (1989).

${ }^{9}$ P. Orlandi, "Vortex dipole rebound from a wall," Phys. Fluids A 2, 1429 (1990).

${ }^{10}$ J. H. G. M. van Geffen, V. V. Meleshko, and G. J. F. van Heijst, "Motion of a two-dimensional monopolar vortex structure in a bounded rectangular domain," Phys. Fluids 8, 2393 (1996).

${ }^{11}$ H. J. H. Clerex, S. R. Maassen, and G. J. F. van Heijst, "Decaying twodimensional turbulence in square containers with no-slip or stress-free boundaries," Phys. Fluids 11, 611 (1999).

${ }^{12}$ H. J. H. Clercx and G. J. F. van Heijst, "Energy spectra for decaying 2D turbulence in a bounded domain," Phys. Rev. Lett. 85, 306 (2000).

${ }^{13}$ H. J. H. Clercx and A. H. Nielsen, "Vortex statistics for turbulence in a container with rigid boundaries," Phys. Rev. Lett. 85, 752 (2000).

${ }^{14} \mathrm{~S}$. Li, D. Montgomery, and W. B. Jones, "Inverse cascades of angular momentum," J. Plasma Phys. 56, 615 (1996).

${ }^{15} \mathrm{~S}$. Li, D. Montgomery, and W. B. Jones, "Two-dimensional turbulence with rigid circular walls," Theor. Comput. Fluid Dyn. 9, 167 (1997).

${ }^{16}$ J. B. Flór, "Coherent vortex structures in stratified fluids," Ph.D. thesis, Eindhoven University of Technology, 1994.

${ }^{17}$ C. T. Yap and C. W. van Atta, "Experimental studies of the development of quasi-two-dimensional turbulence in stably stratified fluid," Dyn. Atmos. Oceans 19, 289 (1993).

${ }^{18}$ A. M. Fincham, T. Maxworthy, and G. R. Spedding, "Energy dissipation and vortex structure in freely decaying, stratified grid turbulence," Dyn. Atmos. Oceans 23, 155 (1996).

${ }^{19}$ J.-T. Lin and Y.-H. Pao, "Wakes in stratified fluids," Annu. Rev. Fluid Mech. 11, 317 (1979).

${ }^{20}$ J. J. Riley, R. W. Metcalfe, and M. A. Weissman, "Direct numerical simulations of homogeneous turbulence in density stratified fluids," in Proceedings of the AIP Conference on Nonlinear Properties of Internal Waves, edited by B. J. West (AIP, New York, 1981), p. 79.

${ }^{21}$ D. K. Lilly, "Stratified turbulence and the mesoscale variability of the atmosphere," J. Atmos. Sci. 40, 749 (1983).

${ }^{22}$ S. R. Maassen, "Self-organization of confined two-dimensional flows," Ph.D. thesis, Eindhoven University of Technology, 2000.

${ }^{23}$ J. M. H. Fortuin, "Theory and application of two supplementary methods of constructing density gradient columns," J. Polym. Sci. 44, 505 (1960).

${ }^{24}$ R. D. Blevins, Applied Fluid Dynamics Handbook (Van Nostrand Reinhold, New York, 1984).

${ }^{25}$ S. Dalziel, DigImage. Image Processing for Fluid Dynamics (Cambridge Environmental Research Consultants Ltd., Cambridge, UK, 1992).

${ }^{26} \mathrm{C}$. W. Clenshaw, "Curve fitting with a digital computer," Comput. J. (UK) 2, 170 (1960)

${ }^{27}$ J. J. Riley and M.-P. Lelong, "Fluid motions in the presence of strong stable stratification," Annu. Rev. Fluid Mech. 32, 613 (2000).

${ }^{28}$ H. J. H. Clercx, A. H. Nielsen, D. J. Torres, and E. A. Coutsias, "Twodimensional turbulence in square and circular domains with no-slip walls," Eur. J. Mech. B/Fluids 20, 557 (2001).

${ }^{29} \mathrm{~J}$. Weiss, "The dynamics of enstrophy transfer in two-dimensional hydrodynamics," Technical Report LJ1-TN-81-121, La Jolla Institute, 1981. 
${ }^{30}$ A. E. Hansen, D. Marteau, and P. Tabeling, "Two-dimensional turbulence and dispersion in a freely decaying system," Phys. Rev. E 58, 7261 (1998).

${ }^{31}$ J. A. van de Konijnenberg, J. B. Flór, and G. J. F. van Heijst, "Decaying quasi-two-dimensional flow on a square domain," Phys. Fluids 10, 595 (1998).

${ }^{32}$ B. M. Boubnov, S. B. Dalziel, and P. F. Linden, "Source-sink turbulence in a stratified fluid," J. Fluid Mech. 261, 273 (1994). 